TRANSACTIONS OF THE

AMERICAN MATHEMATICAL SOCIETY

Volume 352, Number 10, Pages 4421-4450

S 0002-9947(00)02667-2

Article electronically published on June 13, 2000

\title{
SIMPLE ALGEBRAIC AND SEMIALGEBRAIC GROUPS OVER REAL CLOSED FIELDS
}

\author{
YA'ACOV PETERZIL, ANAND PILLAY, AND SERGEI STARCHENKO
}

\begin{abstract}
We continue the investigation of infinite, definably simple groups which are definable in o-minimal structures. In Definably simple groups in o-minimal structures, we showed that every such group is a semialgebraic group over a real closed field. Our main result here, stated in a model theoretic language, is that every such group is either bi-interpretable with an algebraically closed field of characteristic zero (when the group is stable) or with a real closed field (when the group is unstable). It follows that every abstract isomorphism between two unstable groups as above is a composition of a semialgebraic map with a field isomorphism. We discuss connections to theorems of Freudenthal, Borel-Tits and Weisfeiler on automorphisms of real Lie groups and simple algebraic groups over real closed fields.
\end{abstract}

\section{INTRODUCTION}

This is the second of two papers analyzing definably simple groups which are definable in o-minimal structures. These are groups $\mathbb{G}=\langle G, \cdot\rangle$ definable in some o-minimal structure $\mathcal{M}$, which are nonabelian and have no $\mathbb{G}$-definable nontrivial proper normal subgroups.

In the first paper ([14]) we showed that if $\mathbb{G}$ is an infinite such group, then a real closed field $R$ is definable in $\mathcal{M}$ and $G$ is definably isomorphic in $\mathcal{M}$ to a semialgebraic subgroup of $\mathrm{GL}(n, R)$.

The main difference between the two papers is the category in which we are working. While in the first paper we were interested in objects definable in the full structure $\mathcal{M}$ (where, for example, a basis for the topology of $M$ is definable), we investigate now the abstract group structure of $\mathbb{G}$ and objects definable in it. As we show here, there are strong connections between the two categories.

Before we state the main theorem we review some model theoretic definitions. We say that a structure $\mathcal{M}$ is interpretable in a structure $\mathcal{N}$ if $\mathcal{M}$, is isomorphic to a structure whose universe is a quotient of an $\mathcal{N}$-definable set by an $\mathcal{N}$-definable equivalence relation, and its atomic relations are the images of $\mathcal{N}$-definable sets under this quotient (for example, a field of fractions of a given integral domain is interpretable in this domain). If $\mathcal{M}$ and $\mathcal{N}$ are interpretable in each other via the isomorphisms $f$ and $g$, respectively, then the maps $g \circ f$ and $f \circ g$ give in a natural way an interpretation of $\mathcal{M}$ in itself and of $\mathcal{N}$ in itself, respectively. $\mathcal{M}$ and $\mathcal{N}$ are called bi-interpretable if $f$ and $g$ can be chosen so that $g \circ f$ is definable

Received by the editors February 25, 1998.

1991 Mathematics Subject Classification. Primary 03C64, 22E15, 20G20; Secondary 12J15.

We thank the referee for valuable comments.

The second and the third authors were partially supported by NSF.

(C)2000 American Mathematical Society 
in $\mathcal{M}$ and $f \circ g$ is definable in $\mathcal{N}$. This is a very strong form of identification between two structures which are not necessarily in the same language. In general, having the interpretations $f$ and $g$ does not guarantee that $\mathcal{M}$ and $\mathcal{N}$ are indeed bi-interpretable: As is shown in [1, the group of unitriangular matrices over an algebraically closed field $K$ and the field $K$ are interpretable in each other but not bi-interpretable. For more on interpretations and bi-interpretations, see 8 .

We say that an algebraic group defined over $R$ is $R$-simple if it has no nontrivial normal algebraic subgroups defined over $R$. Note that if $R$ is a real closed field, then its algebraic closure $K=R(\sqrt{-1})$ can be identified with $R^{2}$ in a natural way and thus algebraic subgroups of $\operatorname{GL}(n, K)$ can also be viewed as algebraic subgroups of $\mathrm{GL}(2 n, R)$. Our classification of definably simple groups in o-minimal structures needs to take this into account.

The main theorem of this paper is:

Theorem 1.1. Let $\mathbb{G}=\langle G, \cdot\rangle$ be an infinite, definably simple group which is definable in some o-minimal structure. Then there is a real closed field $k$ such that one and only one of the following holds:

1. $\mathbb{G}$ and $\langle k(\sqrt{-1}),+, \cdot\rangle$ are bi-interpretable. $G$ is definably isomorphic, in $\mathbb{G}$, to the $k(\sqrt{-1})$-rational points of a linear algebraic group defined over $k(\sqrt{-1})$.

2. $\mathbb{G}$ and $\langle k,+, \cdot\rangle$ are bi-interpretable. $G$ is definably isomorphic, in $\mathbb{G}$, to the semialgebraic connected component of a group $\hat{G}(k)$, where $\hat{G}$ is a $k$-simple algebraic group defined over $k$.

Remark 1.2. (a) Cases (1) and (2) can also be characterized in model theoretic terms, as in the cases where $\mathbb{G}$ is either stable or unstable, respectively. Since every algebraically closed field of characteristic zero is the algebraic closure of some real closed field, Theorem 1.1 gives a model theoretic characterization of simple algebraic groups over algebraically closed fields of characteristic zero as precisely those stable, simple groups which are definable in some o-minimal structure.

(b) Let $G$ be a semialgebraic group over a real closed field $R$. Then we can define in a natural way its Lie algebra $\mathcal{L}=L(G)$ over $R$ (see [14). If $G$ is definably simple then, as we show here, $G$ does not have semialgebraic normal subgroups, therefore (see [14]) $\mathcal{L}$ is a simple Lie algebra. We let $\mathcal{L}^{\prime}$ be the "complexification" of $\mathcal{L}$, namely the canonical extension of $\mathcal{L}$ to a Lie algebra over $R(\sqrt{-1})$. If $\mathcal{L}^{\prime}$ is simple, then $\mathcal{L}$ is called absolutely simple. As we will show towards the end of this paper, $G$ is bi-interpretable with a real closed field if and only if $\mathcal{L}$ is absolutely simple.

(c) Theorem 1.1 can be seen as a strong version of an o-minimal analogue of Cherlin's Conjecture. This conjecture states that any noncommutative (definably) simple group of finite Morley rank is a linear algebraic group over an algebraically closed field. Theorem 1.1 proves this conjecture for groups of finite Morley rank definable in o-minimal structures and gives the best possible analogue for definably simple groups which are unstable (in particular, not of finite Morley rank) and definable in an o-minimal structure. For more on the current status of general Cherlin's Conjecture see [4].

We recall that if $G$ is a semialgebraic group over a real closed field $R$, then it carries a canonical topology induced from $R$. If $R$ is the field of real numbers, then $G$ is a Lie group. 
The following is a corollary of the second clause of Theorem 1.1 As we prove, it holds when $G$ is replaced with any semialgebraic group which is bi-interpretable with a real closed field.

Corollary 1.3. Let $G$ be an unstable, semialgebraic, definably simple group over a real closed field $R$. Then the following hold:

1. Every semialgebraic subset of $G^{n}$ is $\mathbb{G}$-definable;

2. Let $H$ be any other semialgebraic group over a real closed field $L$. Then every abstract group isomorphism $f: G \rightarrow H$ is of the form $f=g \circ h$ where $h$ is a map induced by an abstract field isomorphism between $R$ and $L$ and $g$ is an $L$-semialgebraic isomorphism of groups. In particular, $f$ is continuous, and if $R=L=$ the field of real numbers, then $f$ is semialgebraic and therefore a Lie isomorphism of $G$ and $H$.

An analogue of the above for simple algebraic groups over any algebraically closed field can be found in Poizat's book on stable groups (see 4.16, 4.17 in [19]).

Remark 1.4. (i) Part (2) of the corollary above is related to a large body of mathematical work on the connections between abstract group homomorphisms of certain algebraic groups (or simple Lie groups) and algebraic homomorphisms (or analytic maps) between these groups. (2) follows, in the $R$-isotropic case, from a theorem of Borel-Tits ([3]) and in the $R$-anisotropic case, from a theorem of Weisfeiler ([22]). However, our treatment is independent of those. Moreover, the bi-interpretability results seem to shed new light on the strong connections between the abstract group structure of $G$ and the structure which $G$ inherits from the ambient field. In [21], $J$. Tits posed in somewhat intuitive terms the following question: Given a (simple) algebraic group $G$ defined over a field $K$, does the group $G(K)$ of $K$-rational points "carry with it the data of the field $K$ and the algebraic structure of $G$ "? Tits' own idea for an answer was given in terms of homomorphisms of such groups. The bi-interpretability theorem suggests, when $K$ is a real closed field, a more explicit answer to this question.

(ii) Since every simple Lie group is isomorphic, as a Lie group, to a semialgebraic linear group, Corollary 1.3 together with Remark (b) preceding it, implies the classical theorem of Freudenthal ([7]) (generalizing a theorem of Cartan, van den Waerden on compact Lie groups): If $G$ is a simple Lie group whose Lie algebra is absolutely simple, then every abstract automorphism of $G$ is analytic.

The paper is divided into two main parts. In the first part we show that a field $K$ is interpretable in the group structure $\mathbb{G}$. By our earlier results, we may assume here that $G$ is a semialgebraic group over a real closed field $R$. We then apply some theory of algebraic groups according to the $R$-isotropic, anisotropic cases, and transfer results from the field of real numbers to an arbitrary real closed field. We make use here of the work on compact groups from [11 and prove an ominimal analogue of a theorem by Zil'ber on the interpretation of fields in solvable, nonnilpotent groups.

In the second part we show that the group $G$ is definably isomorphic in the sense of $\mathbb{G}$ to a linear group over the field $K$ defined above, (or possibly another field replacing $K$ ). We actually make no use here of the fact that $G$ is semialgebraic and develop some general model theoretic machinery, mainly in the general context of geometric structures. 
Now we briefly introduce basic terminology: By "definable" we always mean "definable with parameters". We use the letters $\mathcal{M}, \mathcal{N}, \mathcal{X} \ldots$ to denote different structures and $M, N, X \ldots$ to denote their universes. We use $\mathbb{G}$ to denote the group structure $\langle G, \cdot\rangle$, but sometimes just use $G$ or $H$ to denote certain groups. The structures in question change throughout the paper but we try to make this point clear at the beginning of each section. We remind the reader that an $o$ minimal structure is a densely ordered structure $\mathcal{M}=\langle M,<, \cdots\rangle$ such that every definable subset of $M$ is a finite union of intervals with endpoints in $M \cup\{ \pm \infty\}$. For more on o-minimal structures, we suggest the article and book of L. van den Dries [5], 6].

Definable groups in o-minimal structures have the Descending Chain Condition on definable subgroups. Therefore, every definable group $G$ has a minimal definable subgroup of finite index, denoted by $G^{0}$. If $G=G^{0}$, then $G$ is called $a$ connected group. However, since we refer here to definability with respect to several different structures we will use terminology like "semialgebraically connected" for semialgebraic groups without semialgebraic proper subgroup of finite index, or "algebraically connected" for algebraic groups without proper algebraic subgroups of finite index, etc.

We refer the reader to Section 1.2 in [14 for the basic facts on groups definable in o-minimal structures.

Infinitesimal neighborhoods. Because of the topological setting, many of the properties that we will obtain along the way are local in nature. One way to tackle these issues is to use the notion of an infinitesimal group. This is a notion that is used often, possibly under other names, in the theory of Lie groups. Model Theory allows us to treat it more rigorously:

We start with a given o-minimal structure $\mathcal{N}$ and let $\mathcal{N}^{*}$ be an $|N|^{+}$-saturated elementary extension of $\mathcal{N}$. If $G$ is a definable group in $\mathcal{N}$ we denote by $G^{\mathcal{N}}$ and $G^{\mathcal{N}^{*}}$ the points in $\mathcal{N}$ and $\mathcal{N}^{*}$ which lie in $G$, respectively. For $g \in G^{\mathcal{N}}$, we denote by $\mathcal{V}_{g}$ the infinitesimal neighborhood of $g$ in $\mathcal{N}^{*}$, namely the intersection of all open neighborhoods of $g$ (in the group topology) which are definable in $\mathcal{N}$. By continuity, for $g, h \in G^{\mathcal{N}}, \mathcal{V}_{g} \cdot \mathcal{V}_{h}=\mathcal{V}_{g h}$ (hence $\mathcal{V}_{e}$ is a subgroup of $G^{\mathcal{N}^{*}}$ ), and $\mathcal{V}_{g}^{\mathcal{V}_{h}} \subseteq \mathcal{V}_{g^{h}}$. In the text that follows we use infinitesimal neighborhoods without referring to a particular elementary extension of $\mathcal{N}$. In all cases the results and proofs are independent of $\mathcal{N}^{*}$.

\section{Defining A FIELD From the GROUP}

Our goal in this part of the paper is to show that given $G$ which is infinite, definably simple and definable in some o-minimal structure, one can interpret a field in the group structure $\mathbb{G}$. In [14] we see that such a field is definable in the language of the o-minimal structure, and indeed we will make use of this fact when we come to define the field in $\mathbb{G}$. Before turning to the setting of the main theorem, we develop some general tools for handling certain subgroups, not necessarily definable, of definable groups in o-minimal structures.

\section{1. $\bigvee$-definable subgroups. We work here in an o-minimal structure $\mathcal{N}$.}

Definition 2.1. Let $G$ be a definable group, $\left\{X_{i}: i \in I\right\}$ a collection of definable subsets of $G$. A subgroup $H=\bigcup_{i} X_{i}$ is called $a \bigvee$-definable subgroup of $G$ if the following hold: 
(i) For every $i, j \in I$ there is $k \in I$ such that $X_{i}, X_{j} \subseteq X_{k}$.

(ii) For every $i, j \in I$ there is $k \in I$ such that $X_{i} \cdot X_{j} \subseteq X_{k}$.

(iii) For every $i \in I$ there is $j \in I$ such that $X_{i}^{-1} \subseteq X_{j}$.

If $H$ is a $\bigvee$-definable subgroup of $G$, we denote by $\operatorname{dim} H$ the maximum dimension of the $X_{i}$ 's.

Remark 2.2. We emphasize that the collection of $X_{i}$ 's in the above definition is a fixed collection rather than a definable, parameterized family of sets. This is crucial for the theorem below. Also, since a $\bigvee$-definable subgroup is a union of definable sets its realization in every elementary extension $\mathcal{N}^{*}$ of $\mathcal{N}$ is well defined and is again a subgroup of $G^{\mathcal{N}^{*}}$.

Proposition 2.3. Let $\langle G, \cdot, e\rangle$ be a definable group over a set $B \subseteq N, H=\bigcup_{i \in I} X_{i}$ $a \bigvee$-definable subgroup of $G$, where the $X_{i}$ 's are definable over $B$.

1. There is $i \in I$, call it $i_{0}$, such that $\operatorname{dim} X_{i_{0}}=\operatorname{dim} H$ and $\mathcal{V}_{e} \cap X_{i_{0}}=\mathcal{V}_{e} \cap H$.

2. Let $H_{e}=\mathcal{V}_{e} \cap H$. If $X_{i_{0}} \subseteq X_{j}$ and $g \in \mathcal{N}$ is generic in $X_{j}$ over $B$, then $H_{e}=g^{-1}\left(\mathcal{V}_{g} \cap X_{j}\right)=\left(\mathcal{V}_{g} \cap X_{j}\right) g^{-1}$.

3. If $H_{1} \subset H$ is another $\bigvee$-definable subgroup of $G$ in $\mathcal{N}$, and $\operatorname{dim} H_{1}=\operatorname{dim} H$, then $\mathcal{V}_{e} \cap H_{1}=\mathcal{V}_{e} \cap H$.

Proof. We first claim that under the above assumptions the following holds: For $i_{0} \in I, \mathcal{V}_{e} \cap X_{i_{0}}=\mathcal{V}_{e} \cap H$ if and only if for every $X_{i}$ containing $X_{i_{0}}$ there is (in $\mathcal{N}$ ) a definable open neighborhood $V_{i}$ of $e$ such that $V_{i} \cap X_{i_{0}}=V_{i} \cap X_{i}$.

Indeed, the "right to left" is immediate, since $\mathcal{V}_{e}$ is contained in every $\mathcal{N}$-definable open neighborhood of $e$. As for the opposite direction, consider $\mathcal{N}^{*}$, an $|N|^{+}$saturated elementary extension of $\mathcal{N}$. If the statement on the right hand side fails, then for some $i \in I$, the type $x \in\left(X_{i_{0}} \cap \mathcal{V}_{e}\right) \backslash X_{i}$ can be realized in $\mathcal{N}^{*}$, thus leading to a contradiction.

As the claim shows, we can prove the property $\mathcal{V}_{e} \cap X_{i_{0}}=\mathcal{V}_{e} \cap H$ when the ground model $\mathcal{N}$ is replaced by any elementary extension of it. We can therefore assume that $\mathcal{N}$ is $|B|^{+}$-saturated.

We choose $X_{i}$ of maximal dimension. Now, if $g \in \mathcal{N}$ is generic in $X_{i}$ over $B$, then $g$ is generic over $B$ in every $X_{j}$ containing $X_{i}$ and therefore $\mathcal{V}_{g} \cap X_{i}=\mathcal{V}_{g} \cap X_{j}$. Now take $i_{0}$ such that $X_{i}^{-1} X_{i} \subseteq X_{i_{0}}$ and $i_{1}$ such that $X_{i} X_{i_{0}} \subseteq X_{i_{1}}$. The set $g^{-1}\left(\mathcal{V}_{g} \cap X_{i}\right)$ is a subset of $\mathcal{V}_{e} \cap X_{i_{0}}$ and therefore, after multiplying on the left by $g$, we have

$$
\mathcal{V}_{g} \cap X_{i} \subseteq g\left(\mathcal{V}_{e} \cap X_{i_{0}}\right) \subseteq \mathcal{V}_{g} \cap X_{i_{1}}
$$

But the sets on both ends of the above inclusion are equal, hence $\mathcal{V}_{g} \cap X_{i}=$ $g\left(\mathcal{V}_{e} \cap X_{i_{0}}\right)$, and by symmetry we also have $\mathcal{V}_{g} \cap X_{i}=\left(\mathcal{V}_{e} \cap X_{i_{0}}\right) g$. We may conclude then that

$$
g^{-1}\left(\mathcal{V}_{g} \cap X_{i}\right)=\mathcal{V}_{e} \cap X_{i_{0}}=\left(\mathcal{V}_{g} \cap X_{i}\right) g^{-1} .
$$

The above argument can clearly be repeated when $i_{0}$ is replaced by any $j \geq i_{0}$; therefore,

$$
g^{-1}\left(\mathcal{V}_{g} \cap X_{i}\right)=\mathcal{V}_{e} \cap X_{j}=\left(\mathcal{V}_{g} \cap X_{i}\right) g^{-1}
$$

It follows that $\mathcal{V}_{e} \cap X_{j}=\mathcal{V}_{e} \cap X_{i_{0}}$ for every $X_{j}$ containing $X_{i_{0}}$. (1) and (2) of the proposition clearly follow.

We now take $H_{1}=\bigcup_{j \in J} Y_{j} \subseteq H$ a $\bigvee$-definable subgroup of $G$ with the same dimension as $H$. Without loss of generality, $H_{1}$ is definable over $B$ as well. Let 
$X_{i_{0}}$ and $Y_{j_{0}}$ be as given by (1) for $H$ and $H_{1}$, respectively. It is easy to see that $\mathcal{V}_{e} \cap H=\mathcal{V}_{e} \cap H_{1}$ if and only if there is in $\mathcal{N}$ a definable open neighborhood $V$ of $e$ such that $V \cap X_{i_{0}}=V \cap X_{j_{0}}$. We therefore may assume again that $\mathcal{N}$ is $|B|^{+}$-saturated.

Note that (2) implies that for $g$ generic in $X_{j}$ containing $X_{i_{0}}$,

$$
\mathcal{V}_{e} \cap H=g^{-1}\left(\mathcal{V}_{g} \cap H\right) \text {. }
$$

Let $g$ be generic in $Y_{j_{0}}$ over $B$. Then, since $\operatorname{dim} H=\operatorname{dim} H_{1}, g$ is also generic in any $X_{j}$ containing it. Therefore, $\mathcal{V}_{g} \cap H=\mathcal{V}_{g} \cap H_{1}, \mathcal{V}_{e} \cap H_{1}=g^{-1}\left(\mathcal{V}_{g} \cap H_{1}\right)$ and $\mathcal{V}_{e} \cap H=g^{-1}\left(\mathcal{V}_{g} \cap H\right)$. It follows that $\mathcal{V}_{e} \cap H=\mathcal{V}_{e} \cap H_{1}$.

Note that if $\left\{X_{i}: i \in I\right\}$ is any fixed collection of definable subsets of $G$ over $B$, all containing $e$, then the subgroup of $G$ generated by all the $X_{i}$ 's can be written as a union of a directed collection of sets of the form $A=X_{\alpha(1)}^{\epsilon_{1}} \cdots X_{\alpha(m)}^{\epsilon_{m}}$, for $\alpha(1), \ldots, \alpha(m) \in I$ and $\epsilon_{1}, \ldots, \epsilon_{m} \in\{ \pm 1\}$. This union forms a group in any elementary equivalent structure over $B$, and therefore, as easily seen, it remains a $\bigvee$-definable subgroup of $G$. In general, such subgroups may not be definable. For example, if $R$ is a nonarchimedean real closed field, then the subgroup of $\langle R,+\rangle$ generated by the interval $[-1,1]$ is a $\bigvee$-definable subgroup which is not definable.

The following theorem can be seen as the o-minimal analogue of what is sometimes called Zil'ber's Indecomposability Theorem.

Theorem 2.4. Let $G$ be a definable group, $\left\{X_{i}: i \in I\right\}$ a fixed collection of definable subsets of $G$, all containing $e$. Let $H$ be the subgroup of $G$ generated by the $X_{i}$ 's. Then there are $\alpha(1), \ldots, \alpha(m) \in I$ and $\epsilon_{1}, \ldots, \epsilon_{m} \in\{ \pm 1\}$ such that the set

$$
X=X_{\alpha(1)}^{\epsilon_{1}} \cdots X_{\alpha(m)}^{\epsilon_{m}}
$$

satisfies the following properties:

1. $\operatorname{dim} X=\operatorname{dim} H$.

2. For every open neighborhood $U$ of e there is an open neighborhood $V$ of e such that $(V \cap X) \cdot(V \cap X) \subseteq U \cap X$.

3. $\mathcal{V}_{e} \cap X=\mathcal{V}_{e} \cap H$.

Proof. (1) and (3) follow immediately from Proposition 2.3 and the remarks preceding the theorem. (2) follows from (3) since $\mathcal{V}_{e} \cap X$ is a subgroup of $G$.

In this paper we mainly use the following corollary to the above theorem.

Corollary 2.5. Let $G$ be a definable group, $\left\{X_{i}: i \in I\right\}$ a fixed collection of definable subsets of $G$, all containing $e$. Then there are $\alpha(1), \ldots, \alpha(m) \in I$, $g_{1}, \ldots, g_{m} \in G$ and $\epsilon_{1}, \ldots, \epsilon_{m} \in\{ \pm 1\}$ such that

$$
\mathcal{V}_{e} \cap\left(X_{\alpha(1)}^{\epsilon_{1}}\right)^{g_{1}} \cdots\left(X_{\alpha(m)}^{\epsilon_{m}}\right)^{g_{m}}
$$

is a normal subgroup of $\mathcal{V}_{e}$.

Proof. Consider the collection $\left\{X_{i}^{g}: g \in G^{\mathcal{N}}\right\}$ and the subgroup of $G$, call it $H$, which is generated by this collection in $\mathcal{N}$. Note that $H$ is invariant under conjugation by elements of $G^{\mathcal{N}}$. It is a $\bigvee$-definable group and by Theorem 2.4 there are $\alpha(1), \ldots, \alpha(m) \in I, g_{1}, \ldots, g_{m} \in G^{\mathcal{N}}$ and $\epsilon_{1}, \ldots, \epsilon_{m} \in\{ \pm 1\}$ such that for $X=\left(X_{\alpha(1)}^{\epsilon_{1}}\right)^{g_{1}} \cdots\left(X_{\alpha(m)}^{\epsilon_{m}}\right)^{g_{m}}$, we have $\mathcal{V}_{e} \cap X=\mathcal{V}_{e} \cap H$.

As was claimed in the proof of [2.3, it follows that for every $g$ (in $G^{\mathcal{N}}$ ) there is a definable neighborhood $V(g)$ of $e$ (in $\mathcal{N}$ ) such that $V(g) \cap X=V(g) \cap X^{g}$. This is 
now a first order property of $\mathcal{N}$ which remains true in every elementary extension of $\mathcal{N}$.

We now claim that for some $g_{0} \in G$ there are definable neighborhoods $W_{0}$ of $g_{0}$ and $V_{0}$ of $e$ such that for every $g \in W_{0}$,

$$
V_{0} \cap X=V_{0} \cap X^{g} .
$$

Indeed, since this is a first order property, we may assume that $\mathcal{N}$ is saturated over the parameters defining $X$. If $g_{0}$ generic is in $G$, then we can find a neighborhood $V\left(g_{0}\right)$ of $e$ such that $V\left(g_{0}\right) \cap X=V\left(g_{0}\right) \cap X^{g_{0}}$. Moreover, by shrinking $V\left(g_{0}\right)$ if needed, we may assume that $g_{0}$ is still generic over the parameters defining $V\left(g_{0}\right)$. By genericity it now follows that for some neighborhood $W_{0}$ of $g_{0}$, for all $g \in W_{0}, V\left(g_{0}\right) \cap X=V\left(g_{0}\right) \cap X^{g}$, thus proving the claim.

We now take $g_{0}$ in $G^{\mathcal{N}}, W_{0}$ and $V_{0} \mathcal{N}$-definable, all as in the last claim. Then for all $g \in W_{0}$ we have $V_{0} \cap X=V_{0} \cap X^{g}$, and in particular (since $\mathcal{V}_{e} \subseteq V_{0}$ ), for all $g \in W_{0}$,

$$
\mathcal{V}_{e} \cap X=\mathcal{V}_{e} \cap X^{g}
$$

To prove that $\mathcal{V}_{e} \cap X$ is normal in $\mathcal{V}_{e}$, we take arbitrary $h \in \mathcal{V}_{e}$ and conjugate both sides of the above equality for the case $g=g_{0}$. $h$ is of the form $g_{0}^{-1} g$ for some $g \in W_{0}$, therefore (applying the above again for $g$ ) we have

$$
\left(\mathcal{V}_{e} \cap X\right)^{h}=\left(\mathcal{V}_{e} \cap X^{g_{0}}\right)^{h}=\mathcal{V}_{e}^{h} \cap X^{g_{0} h}=\mathcal{V}_{e} \cap X^{g}=\mathcal{V}_{e} \cap X
$$

2.2. Defining a field in a solvable nonnilpotent group. In this section $\mathcal{N}$ is an o-minimal structure with definable choice functions. Any o-minimal expansion of an ordered group is such. We need definable choice functions in order to replace any group interpretable in $\mathcal{N}$ with a definable one.

The following theorem is an o-minimal version of a theorem by Zil'ber (see 3.7 in [19]). Given a definable group $A$, we say that another definable group $M$ is $a$ definable group of automorphisms of $A$ if $M$ acts on $A$ as a group of automorphisms and the map $(\alpha, a) \mapsto \alpha(a)$ from $M \times A$ into $A$ is definable.

Theorem 2.6. Let $A$ be a definable abelian group, $M$ an infinite definable abelian group of distinct automorphisms of $A$. If $A$ is $M$-minimal (i.e. there is no infinite definable subgroup of $A$ invariant under $M)$, then there is a definable infinite field $K$, such that $A$ is definably isomorphic to $\langle K,+\rangle$ and $M$ is definably isomorphic to a subgroup of $K^{\times}$. Moreover, all the definable objects above are definable in the structure containing the groups $M, A$ and the action of $M$ on $A$.

Proof. Unless otherwise stated we take all definability to be in the structure containing the groups and their action. Since the group action is definable in $\mathcal{N}$ it is easily seen to be continuous with respect to the o-minimal group topology on $M$ and $A$ and the product topology on $M \times A$ (see for example the proof in [12] that the topology of the underlying additive group of a definable ring makes ringmultiplication continuous as well).

We denote by 0 the identity element of $A$. For $a \in A$ and $m \in M$, we let $m a$ denote the action of $m$ on $a$.

For every $n$, we consider the set $M^{2 n}$ as a set of endomorphisms of $A$ as follows: Given $\bar{m}=\left(m_{1}, \ldots, m_{2 n}\right) \in M^{2 n}$ and $a \in A$,

$$
\bar{m} a=\left(m_{1} a-m_{2} a\right)+\ldots+\left(m_{2 n-1} a-m_{2 n} a\right) .
$$


We let $\sim$ be the equivalence relation on $\bar{M}=\bigcup_{l \in \mathbb{N}} M^{2 l}$ which identifies equal endomorphisms. We denote by $[\bar{m}]$ the equivalence class of $\bar{m}$, and identify it with the corresponding endomorphism of $A$. We also use $\alpha, \beta$ to denote these endomorphisms. The set $R=\bar{M} / \sim$ is easily seen to be a ring of definable endomorphisms of $A$ with respect to addition and composition of maps.

Lemma 2.7. Every nonzero $\alpha \in R$ is an automorphism of $A$.

Proof. Notice that by the commutativity of $M, \operatorname{ker}(\alpha)$ is invariant under $M$, hence since $A$ is minimal, $\operatorname{ker}(\alpha)$ is finite. But then $\operatorname{dim} \alpha A=\operatorname{dim} A$, and again $\alpha A$ is invariant under $M$ and therefore $\alpha$ is surjective. It is thus left to show if $\alpha$ is nonzero, then its kernel must be trivial.

Since every nonzero $\alpha$ is surjective, if $\operatorname{ker}(\alpha)$ is nontrivial, then we get an increasing chain of finite subgroups, all invariant under $M$,

$$
\operatorname{ker}(\alpha) \subsetneq \operatorname{ker}\left(\alpha^{2}\right) \cdots \subsetneq \operatorname{ker}\left(\alpha^{n}\right) \subsetneq \cdots .
$$

As we now show, there are at most finitely many torsion elements in $A$, contradicting the above.

Claim 2.8. Given $a \in A$, the following are equivalent:

(i) $a$ is a torsion element of $A$.

(ii) There is $m \neq 1$ in $M$ such that $m a=a$.

(iii) There is a nonzero $\alpha \in R$ such that $\alpha a=0$.

Proof. Assume that $n a=0 . A^{\prime}$, the collection of all elements in $A$ whose order is at most $n$ forms a definable (abelian) subgroup of $A$ of bounded exponent and therefore it is finite (see [20]). $A^{\prime}$ is invariant under $M$; therefore, the orbit of $a$ under $M$ is finite and thus $\operatorname{Stab}(a)$ is a definable subgroup of $M$ of finite index. In particular, there is $m \neq 1$ in $M$ such that $m a=a$, thus (ii) follows from (i).

Clearly, if $m a=a$, then $(m-1) a=0$ and $m-1 \in R$. Therefore, (ii) implies (iii). We already pointed out that (iii) implies (i).

By the last claim, the subgroup of all torsion elements in $A$ is definable and therefore must be finite.

Notice that the proof of the lemma shows that $A$ is torsion-free. It follows from the lemma that $R$ is an integral domain. Clearly, $R$ itself may not be definable but, as we will show, its field of fractions is definable.

We now fix a nonzero $a \in A^{\mathcal{N}}$ and consider for every $n$, the subset of $A, A_{n}=$ $M^{2 n} a$. The set $H=R a=\bigcup_{n} A_{n}$ is a $\bigvee$-definable subgroup of $A$. Hence, by Proposition 2.3 there is $n_{0}$ such that $\mathcal{V}_{0} \cap A_{n_{0}}=\mathcal{V}_{0} \cap H$, call this set $H_{0}$. For every $n \geq n_{0}$ and every $b$ generic in $A_{n}$ we have $H_{0}=\left(\mathcal{V}_{b} \cap A_{n}\right)-b$.

Given $\alpha \in R, \alpha$ sends $H$ into $H$. However, since $\alpha R$ could be a proper subset of $R, \alpha H$ does not necessarily equal $H$.

Claim 2.9. (i) For every nonzero $\alpha \in R^{\mathcal{N}}, \alpha H_{0}=H_{0}$.

(ii) For every nonzero $\alpha \in R$, the restriction of $\alpha$ to $H$ is an open map from $H$ into $H$.

Proof. Given a nonzero $\alpha \in R^{\mathcal{N}}, \alpha H$ is a subgroup of $H$ which itself is a $\bigvee$ definable subgroup of $A$. Moreover, since $\alpha$ is injective $\operatorname{dim} \alpha H=\operatorname{dim} H$ and hence by Proposition 2.3. $(\alpha H) \cap \mathcal{V}_{0}=H_{0}$. By the continuity of $\alpha$ and $\alpha^{-1}$ and the fact 
that $\alpha \in \mathcal{N}$, we have $\alpha \mathcal{V}_{0}=\alpha^{-1} \mathcal{V}_{0}=\mathcal{V}_{0}$, and therefore $\alpha H_{0}=\alpha\left(H \cap \mathcal{V}_{0}\right)=H_{0}$, thus proving (i).

Note that since 0 is an interior point of $A_{n_{0}}$ in $H$, we may conclude from (i) that for every $n$ and every nonzero $\alpha \in M^{\mathcal{N}^{2 n}} / \sim, 0$ is an interior point of $\alpha A_{n_{0}}$ in $A_{n_{0}}$. This now is a first order property in the sense of $\mathcal{N}$ which holds for every $\alpha \in M^{2 n}$. But then, for every nonzero $\alpha \in R, 0$ is an interior point of $\alpha H$ in $H$. Since $H$ is a topological group, $\alpha \mid H$ is an open map.

Claim 2.10. For every nonzero $\alpha_{1}, \alpha_{2} \in R$, there are nonzero $\beta_{1}, \beta_{2} \in M^{2 n_{0}} / \sim$ such that $\alpha_{1} \beta_{2}=\alpha_{2} \beta_{1}$.

Proof. We let $R_{1}=\left\{\alpha \in R: \alpha a \in H_{0}\right\} . R_{1} a=H_{0} \subseteq M^{2 n_{0}} a$ and since every definable automorphism in $R$ is determined by its value at $a$ we have $R_{1} \subseteq M^{2 n_{0}} / \sim$.

If we now take nonzero $\alpha_{1}, \alpha_{2} \in R$, then, since $\alpha_{1} H_{0}$ and $\alpha_{2} H_{0}$ both contain open sets around 0 , we have

$$
\alpha_{1} H_{0} \cap \alpha_{2} H_{0}=\alpha_{1}\left(R_{1} a\right) \cap \alpha_{2}\left(R_{1} a\right) \neq\{0\} .
$$

Therefore, there are nonzero $\beta_{1}, \beta_{2} \in R_{1} \subseteq M^{2 n_{0}} / \sim$ such that $\alpha_{1} \beta_{1} a=\alpha_{2} \beta_{2} a$ and hence $\alpha_{1} \beta_{1}=\alpha_{2} \beta_{2}$ in $R$.

We now consider the field of fractions of $R$,

$$
K=\{\alpha / \beta: \beta \neq 0, \alpha, \beta \in R\},
$$

defined in the usual way. Even though $K$ is not a-priori definable, or even interpretable, by the above claim it is sufficient to consider all $\alpha / \beta$ such that $\alpha, \beta \in$ $M^{2 n_{0}} / \sim$, thus $K$ is indeed an interpretable field. Moreover, $K$ can be realized as a field of definable automorphisms of $A$ by letting $\alpha / \beta$ be the map $\alpha \beta^{-1}$.

The map $\alpha / \beta \mapsto \alpha / \beta(a)$ is a definable embedding of $\langle K,+\rangle$ into $\langle A,+\rangle$ hence, by the minimality of $A$, its image equals $A$. If we fix $\alpha=\left[\left(m_{1}, \ldots, m_{2 n_{0}}\right)\right]$ and $\beta$, then the map $d \mapsto\left[\left(d m_{1}, \ldots, d m_{2 n_{0}}\right)\right] / \beta$ is an embedding of $M$ into the multiplicative group of $K$. Thus, Theorem 2.6 is proved.

Before we prove the final proposition we need a small group theoretic fact.

Fact 2.11. Let $N$ be a finite normal subgroup of a group $H$ and assume that $H$ has no $\langle H, \cdot\rangle$-definable subgroups of finite index.

(i) If $g \in H$ and $g N$ is in $Z(H / N)$, then $g \in Z(H)$. In particular, $N \subseteq Z(H)$.

(ii) If $N=Z(H)$, then $H / N$ is centerless and has no normal finite subgroups.

Proof. (i) Assume that $g N \subseteq Z(H / N)$. Then the map $h \mapsto h g h^{-1} g^{-1}$ takes elements from $H$ into $N$. This map induces an injection from $H / C_{H}(g)$ into $N$, hence $C_{H}(g)$ has finite index in $H$, thus is equal to $H$. (ii) follows from (i).

Theorem 2.12. Let $G$ be a definable infinite group in an o-minimal structure which is solvable and not nilpotent-by-finite. Then a field $K$ is interpretable in $\mathbb{G}=\langle G, \cdot\rangle$; its additive group is definably isomorphic to a quotient of two definable subgroups of $G$.

Proof. We choose $H$ of minimal positive dimension which is solvable, not nilpotentby-finite, and can be obtained as the quotient of two $\mathbb{G}$-definable subgroups of $G$. We may assume that $H$ has no $\mathbb{G}$-definable subgroups of finite index. Since $H$ is solvable and not nilpotent so is $H / Z(H)$ and therefore by minimality, $Z(H)$ is 
finite. By replacing $H$ with $H / Z(H)$ we may assume (see Fact 2.11) that $H$ is centerless and has no normal finite subgroups.

We now take $K$ to be a definable normal proper subgroup of $H$ of maximal dimension. We claim that $K_{1}=H / K$ is abelian. If not, then since $K_{1}$ is solvable it has an abelian normal subgroup $E$; if $E$ is infinite, then $E_{1}=Z\left(C_{K_{1}}(E)\right)$ is a definable abelian proper subgroup of $K_{1}$ containing $E$. The extension of $K$ by $E_{1}$ is a normal proper subgroup of $H$ which contradicts our choice of $K$. If $E$ is finite, then we replace $K_{1}$ by $K_{1} / E$ and repeat the same argument.

By the minimality of $H, K$ is nilpotent and therefore $Z(K)$ is nontrivial and normal in $H$. But $H$ has no finite normal subgroups so $Z(K)$ is infinite. We take $A \subseteq Z(K)$ to be a minimal $\mathbb{G}$-definablenormal subgroup of $H$ of positive dimension and let $H_{1}=C_{H}(A)$, a normal $\mathbb{G}$-definablesubgroup of $H$ containing $K$. Since $H$ is centerless and has no definable subgroups of finite index, $M=H / H_{1}$ is infinite. It is an infinite abelian group of distinct automorphisms of $A$ (acting by conjugation) and, since every $M$-invariant subgroup of $A$ is clearly normal in $H, A$ is $M$-minimal. Applying Theorem 2.6 we get a field interpretable in $\mathbb{G}$.

2.3. Defining a field in a definably simple group. We return now to the setting of Theorem 1.1] We have a real closed field $R$, and $G$ a semialgebraic subgroup of $\mathrm{GL}(n, R)$. We let $\mathbb{G}=\langle G, \cdot\rangle$ and $\mathcal{R}=\langle R,<,+, *\rangle$.

Our assumption is that $\mathbb{G}$ is an infinite, nonabelian group which has no $\mathbb{G}$ definable normal nontrivial proper subgroups. We want to show that a field is interpretable in $\mathbb{G}$.

We let $K$ be the algebraic closure of $R$, i.e. $K=R(\sqrt{-1})$, and denote by $\bar{G}$ the Zariski closure of $G$ in $\operatorname{GL}(n, K) . \bar{G}$ might not be connected as an algebraic group and we let $H$ be its minimal algebraic subgroup of finite index. Note that $\bar{G}$ and $H$ (which we identify with $\bar{G}(K)$ and $H(K)$ ) are both defined over $R$.

We first state some generalities on algebraic groups.

Fact 2.13. (i) If $k$ is a field of characteristic 0 and $H$ is a connected linear algebraic group defined over $k$, then $H(k)$ is Zariski-dense in $H$.

(ii) Assume that $G$ is an algebraic subgroup of $G L(n, K)$. If $G$ has no $\mathbb{G}$-definable subgroups of finite index, then it has no subgroups of finite index at all.

Proof. For (i) see [2], AG.13.3. (ii) is actually true for all groups of finite Morley rank which are definable in o-minimal structures: If $G$ is abelian, then for every $n$, the group $n G$ has finite index in $G$ (since, as we noted already, a definable infinite group has an unbounded exponent). Therefore, every subgroup of finite index is contained in a definable one.

Assume that $G$ is not abelian and $H$ is a subgroup of $G$ of finite index. By Zil'ber's Indecomposability theorem for groups of finite Morley rank (see 2.9 and the proof of 2.11 in [19]) one can obtain a $\mathbb{G}$-definable $G_{1} \subseteq H$ which is normal in $G$ such that $H / G_{1}$ is central in $G / G_{1}$. It follows that $Z\left(G / G_{1}\right)$ has finite index in $G / G_{1}$ so its pull-back in $G$ is $\mathbb{G}$-definable of finite index and therefore, is equal to $G$. Thus $G / G_{1}$ is abelian so we can apply to it the previous argument.

Definition 2.14. Let $H$ be a connected linear algebraic group defined over a field $k$ of characteristic zero. $H$ is said to be $k$-isotropic if there is some $k$-defined algebraic subgroup $T$ of $H$ which is rationally isomorphic over $k$ to some nontrivial product of copies of the multiplicative group of the field $G_{m}$; such a $T$ is called $a k$-split torus. If $H$ is not $k$-isotropic, it is said to be anisotropic over $k$, or $k$-anisotropic. 
Remark 2.15. Suppose we are working in GL( $n)$. The canonical copies of algebraic tori are diagonal groups where certain entries are 1 and other entries are free to range over nonzero elements. These are defined over $\mathbb{Q}$ and we call them standard tori. Then, (see [2], 8.2), $H$ is $k$-isotropic iff there is $a \in \mathrm{GL}(n, k)$ and some standard torus $T$ such that $T^{a}<H$, equivalently $T(k)^{a}<H(k)$.

The proof that $G$ interprets a field goes via two cases, depending on whether $H$, the algebraic connected component of $\bar{G}$, is $R$-isotropic or $R$-anisotropic. It suggests three categories for a definably simple semialgebraic group $G$ over a real closed field $R$. When $H$ is $R$-isotropic, $G$ is bi-interpretable with an algebraically closed field (e.g. $\operatorname{PSL}(2, R(\sqrt{-1}))$ ), or with a real closed field (e.g. $\operatorname{PSL}(2, R))$. When $G$ is $R$-anisotropic it is necessarily bi-interpretable with a real closed field (e.g. $\mathrm{SO}(3, R))$.

2.3.1. The $R$-anisotropic case. The main idea of this section is to transfer results and arguments from the field of reals to the field $\mathrm{R}$. To do so we first observe some general facts about o-minimal structures.

We assume the following claim to be known and add a proof for the sake of completeness.

Claim 2.16. Let $T$ be an o-minimal theory. Given a formula $\phi(x, y)$ in the language of $T$ there is a formula $\psi(y)$ such that for every model $\mathcal{M}$ of $T, \mathcal{M} \models \psi(a)$ if and only if $\phi(x, a)$ defines a definably connected set.

Proof. We first consider a cell decomposition $C_{1}, \cdots, C_{n}$ of the set defined by $\phi(x, y)$, such that for every $y=a$ and every cell $C_{i}$, the fiber $\left(C_{i}\right)_{a}$ is either a cell or an empty set. We note also that if $A, B$ are two definably connected sets, then $A \cup B$ is definably connected if and only if either $A \cap \bar{B}$ or $\bar{A} \cap B$ are nonempty. Using this fact and the above cell decomposition it is easy to find $\psi(y)$ as needed.

Using a similar argument to the above, one can prove:

Claim 2.17. Let $\mathcal{M}$ be an o-minimal structure, $S$ a definable set, and let $\left\{G_{a}: a \in\right.$ $S$ \} be a uniformly definable family of definable groups (namely, the underlying sets of the groups and the operations are given uniformly). Then the family $\left\{G_{a}^{\circ}: a \in\right.$ $S\}$, of the $\mathcal{M}$-definably connected components of $e$ in these groups, is uniformly definable in $\mathcal{M}$.

Definition 2.18. An algebraic subgroup $N$ of $\operatorname{GL}(n, K)$ is called unipotent if every $g \in N$ is a unipotent element, namely $g-I$ is a nilpotent ring-element in $M(n, K)$. (In particular, then, $(g-I)^{n}=0$.)

A linear algebraic group, algebraically connected, is called reductive if it contains no normal abelian unipotent subgroups.

The following is basically $4.8 \mathrm{in}[2]$.

Fact 2.19. Let $N$ be an algebraic subgroup of $G L(n, K)$. Then

(i) $N$ is unipotent if and only if there is $g \in G L(n, K)$ such that $g^{-1} N g$ is a group of upper triangular matrices with 1 on the diagonal.

(ii) If $N$ is unipotent, then every element of $N$ has an infinite order. In particular, $N$ is infinite.

(iii) $N$ is a nilpotent group. 
Claim 2.20. Let $\left\{H_{a}: a \in S\right\}$ be an $\mathcal{R}$-definable family of algebraic subgroups of $\mathrm{GL}(n, K)$. Then the following hold.

(i) The set of $a$ 's for which $H_{a}$ is a connected, reductive algebraic group is $\mathcal{R}$ definable.

(ii) The set of $a$ 's for which $H_{a}$ is $R$-isotropic is $\mathcal{R}$-definable.

Proof. Since $K=R(\sqrt{-1})$, it can be identified with $R^{2}$ in the usual way, and so every algebraic set in $K^{n}$ is identified with a semialgebraic subset of $R^{2 n}$. By 2.13(ii) an algebraic group over $K$ is connected as an algebraic group if and only if it is semialgebraically connected with respect to $R$. By Claim 2.16 we may assume that for all $a \in S, H_{a}$ is algebraically connected. As pointed out above, an element $g \in H_{a} \subseteq G L(n, K)$ is unipotent if and only if $(g-I)^{n}=0$ and therefore the collection of unipotent elements in $H_{a}$ is uniformly (in $a$ ) definable.

Note first that for every $a \in S$ and every $h \in H_{a}$, if $h$ is not in the center of $H_{a}$, then $h^{H_{a}}$ is infinite. Therefore, by Zil'ber's Indecomposability theorem for groups of finite Morley rank (see [19], 2.9) there is $k \leq 2 n$ such that the $k$-product $\left(h^{H_{a}} h^{-1}\right) \cdots\left(h^{H_{a}} h^{-1}\right)$ is a normal subgroup of $H_{a}$ (possibly not a proper one).

On the other hand, the collection of all unipotent elements of $Z\left(H_{a}\right)$, if any, forms a normal abelian unipotent subgroup of $H_{a}$ (see [2], 4.4(4) and 4.5).

Putting these two observations together we see that $H_{a}$ is reductive if and only if every central element in $H_{a}$ is not unipotent and for all $h \notin Z\left(H_{a}\right)$, the group $\left(h^{H_{a}} h^{-1}\right) \cdots\left(h^{H_{a}} h^{-1}\right)$ is not a unipotent abelian subgroup of $H_{a}$. By the previous comments this is a first order condition on $a$.

(ii) follows immediately from the remarks regarding $k$-isotropic groups and the fact that $R$ is an $\mathcal{R}$-definable subset of $K$.

The following fact, for the field $\mathbb{R}$, is proved in Borel's book ([2], 24.6(c)). By the last claim, that result can be transferred to an arbitrary real closed field.

Fact 2.21. Let $H$ be a (connected) reductive linear algebraic group over a real closed field $R$. $H$ is $R$-anisotropic if and only if $H(R)$ is closed and bounded in $\mathrm{GL}(n, R)$.

We now return to the main theorem, where $G$ is a semialgebraic infinite nonabelian group, with no $\mathbb{G}$-definable normal subgroups, $H$ the algebraic connected component of its Zariski closure, $\bar{G}$, and we assume that $H$ is $R$-anisotropic.

Claim 2.22. $G$ is closed and bounded in $\operatorname{GL}(n, R)$.

Proof. In order to apply 2.21 we will first show that $H$ is reductive.

Notice that $\bar{G}(R)$ contains both $H(R)$ and $G$ and all have the same semialgebraic dimension. It follows that $H_{1}=H(R) \cap G$ has finite index in both $G$ and in $H_{1}$

Let $N$ be the unipotent radical of $H$. That is, $N$ is a unipotent normal connected algebraic subgroup of $H$ which contains all other such subgroups (see 11.21 in [2]). Since $H$ is defined over $R$ and $N$, by maximality, it is fixed by all automorphisms of $H$, the group $N$ is also defined over $R$.

Now, if $H$ is not reductive, then $N$ is nontrivial and therefore (2.19) it is an infinite nilpotent group. By 2.13 (i) $N(R)$ is still an infinite group; it is also normal in $H(R)$. Since $H_{1}=H(R) \cap G$ has finite index in $G$, the group $N_{1}=N(R) \cap G$ is a normal infinite nilpotent subgroup of $H_{1}$. We let $N_{2}=Z\left(N_{1}\right)$, an abelian normal subgroup of $H_{1}$ which is unipotent and therefore infinite. 
If we now let $N_{3}=Z\left(C_{G}\left(N_{2}\right)\right)$, then $N_{2}$ is definable in $\mathbb{G}$, abelian and contains $N_{2}$. Furthermore, it is normalized by $H_{1}$, hence $N_{G}\left(N_{3}\right)$, which is $\mathbb{G}$-definable is a group of finite index in $G$ which therefore equals $G$. It follows that $N_{3}$ is a $\mathbb{G}$ definable normal infinite, abelian subgroup of $G$, contradiction. Hence, $H(R)$ is indeed reductive.

By Fact 2.21 $H(R)$ and hence $H(R) \cap G$ is closed and bounded, and so $G$ is closed and bounded.

One of the main difficulties in the arguments above was the fact that $G$ is not known yet to be semialgebraically connected. We are now ready to prove that.

Below, we let $G^{\circ}$ denote the semialgebraic-connected-component of $e$ in $G$.

Claim 2.23. $G$ is semialgebraically connected, namely $G=G^{\circ}$.

Proof. It is a known fact that if $\hat{G}$ is a compact Lie group over $\mathbb{R}$ and $\hat{T}$ is a maximal abelian subgroup of $\hat{G}$, then

$$
\hat{G}^{\circ}=\bigcup_{g \in G} g \hat{T}^{\circ} g^{-1},
$$

where $\hat{G}^{\circ}$ and $\hat{T}^{\circ}$ are the connected components of $e$ in $\hat{G}$ and $\hat{T}$, respectively $\left(\hat{T}^{\circ}\right.$ is a maximal topological torus in $\hat{G}$ ).

We claim that (3) can be transferred now to any real closed field, when we consider linear semialgebraic $\hat{G}$ and $\hat{T}$ and when we replace the notion of "connected" by "definably connected". To see that, we use Claims 2.16] and 2.17 together with the fact that $\hat{T}$ is a maximal abelian subgroup of $\hat{G}$ if and only if $Z\left(C_{\hat{G}}(\hat{T})\right)=\hat{T}$.

If we now return to our $G$ and let $T$ be a maximal abelian subgroup of $G$ (there is one by Zorn's Lemma), then $Z\left(C_{G}(T)\right)=T$, therefore, $T$ is $\mathbb{G}$-definable. By 2.22 and (3), we see that $G^{\circ}=\bigcup_{g \in G} g T^{\circ} g^{-1}$. But since $T$ is abelian its definably connected component $T^{\circ}$ equals $n T$ for some $n$ hence it is $\mathbb{G}$-definable as well, and therefore $G^{\circ}$ is $\mathbb{G}$-definable. $G^{\circ}$ is of finite index in $G$, therefore $G=G^{\circ}$.

Notice that one corollary of (3) above is that every $g \in G$ is contained in an infinite abelian subgroup of $G$ and thus $C_{G}(g)$ is infinite. We will make use of that later on.

Remark 2.24. As Corollary 1.3 will eventually show, when $\mathbb{G}$ is bi-interpretable with a real closed field, every semialgebraic subset of $G$ is $\mathbb{G}$-definable, therefore $G$ is semialgebraically simple and connected in this case. When $\mathbb{G}$ is bi-interpretable with an algebraically closed field, then it is a group of finite Morley rank and hence its definable simplicity implies that it is abstractly simple (see [19]). In particular, it cannot have any semialgebraic subgroups of finite index. Thus, in both cases our final result implies that $\mathbb{G}$ is semialgebraically simple and connected.

In [11] Nesin and the second author analyze the model theory of compact Lie groups. Our plan is to transfer some theorems and proofs from there to any closed and bounded semialgebraic group. We do so in a sequence of lemmas.

Lemma 2.25. Let $R$ be a real closed field. Assume that $H_{1}, H_{2}$ are semialgebraic closed and bounded subgroups of $\mathrm{GL}(n, R), H_{2}$ normal in $H_{1}$ and $H_{1}$ is semialgebraically connected. Assume further that $B=H_{1} / H_{2}$ is an infinite centerless group such that for every $a \in B, C_{B}(a)^{\circ}$, the semialgebraic connected component of the centralizer of a is abelian, denoted by $T(a)$. Then 
(i) $B$ is covered by the $T(a)$ 's and all are conjugate to each other.

For every $a \in B$,

(ii) $C_{B}(T(a))=T(a)$ and $\left|N_{B}(T(a)) / T(a)\right|=2$.

(iii) $N_{B}(T(a))-T(a)$ contains an involution, which acts on $T(a)$ as $x \mapsto x^{-1}$.

(iv) $T(a)$ has a unique involution and $\operatorname{dim} T(a)=1$.

Proof. The lemma is basically Claims 1-4 from [11], in the proof of Proposition 1.14 there. The assumptions on $B$ in the lemma are exactly those which were used in [11] to prove these claims, thus the lemma is proved there in the case where $R$ is the field of real numbers.

We now claim that it holds in any real closed field. In order to see that the lemma can be transferred to any real closed field we need to verify that all the notions mentioned in it are first-order expressible in the field language. By claims 2.16 and 2.17 one can formulate the assumptions of the modified lemma in a first order way (i.e. as a first order property of the parameters used to define $H_{1}$ and $H_{2}$ ). The conclusions of the lemma are clearly first order expressible, therefore the lemma indeed holds in any real closed field.

Lemma 2.26. Let $R$ be a real closed field. If $B=H_{1} / H_{2}$ is a quotient of two linear semialgebraic groups over $R$ and it satisfies the assumptions of Lemma 2.25, then a real closed field is definable in $\langle B, \cdot\rangle$.

Proof. Notice that since $T(a)=C_{B}(a)^{\circ}$ is abelian it is contained in an abelian group $A>C_{B}(a)$ which is definable in the group language of $B$ (i.e. the center of centralizer of $T(a)$ in $\left.C_{B}(a)\right)$ and has the same dimension as $T(a)$. But then there is $n$ such that $n \cdot A=T(a)$ and therefore $T(a)$ is $\mathbb{G}$-definable. We can then use (i)-(iv), just like Claims 1-4 are used in the proof of Proposition 1.14 in [11] to define a real closed field in the group language of $B$.

In $\mathbb{R}$, every connected compact Lie group is an almost direct product of its center and its derived group. However, since we do not have a way to define in $\mathcal{R}$ the derived group in general, we cannot transfer this fact to an arbitrary real closed field. Instead, we will use the following.

Lemma 2.27. Let $R$ be a real closed field.

(i) If $\left\{G_{a}: a \in U\right\}$ is a semialgebraic family of groups, then the collection of a's for which $G_{a}$ is nilpotent and semialgebraically connected is semialgebraic.

(ii) Let $H_{1}, H_{2}$ be semialgebraic linear groups over $R$ which are closed and bounded. If $G=H_{1} / H_{2}$ is semialgebraically connected nilpotent, then it is abelian.

Proof. We may assume that for all $a, G_{a}$ is semialgebraically connected of dimension at most $n$.

For (i), it is sufficient to show that a definably connected group $G$ of dimension $n$ is nilpotent if and only if $Z_{n}(G)=G$ (where $Z_{n}(G)$ is the $n$-th centralizer in the upper central series of $G$ ).

By dimension considerations there is a first $i \leq n$ such that the dimension of $Z_{i+1}(G)$ equals $\operatorname{dim} Z_{i}(G)$. It follows that $G / Z_{i}(G)$ has finite center and therefore, by Fact 2.11 either $G=Z_{i}(G)$ in which case $G$ is nilpotent and $G=Z_{n}(G)$, or $G / Z_{i+1}(G)$ is infinite and centerless hence $G$ is not nilpotent. Thus (i) is proved.

Since any compact connected nilpotent Lie group is abelian, (ii) holds when $R$ is the field of real numbers. We can use (i) in order to transfer this result to an arbitrary real closed field. 
Proposition 2.28. Let $R$ be a real closed field, $G$ an infinite semialgebraic subgroup of $\operatorname{GL}(n, R)$ which is closed and bounded. If $G$ is not abelian-by-finite, then a real closed field is definable in the group structure $\mathbb{G}$.

Proof. By Lemma 2.26 it is sufficient then to show that one can define in $\mathbb{G}$ two subgroups of $G, H_{1}$ and $H_{2}$, which satisfy the assumptions of Lemma 2.25.

By a $\mathbb{G}$-definable section of $G$ we mean a group of the form $B=H_{1} / H_{2}$ where $H_{i}$ are $\mathbb{G}$-definable. Choose such a definable section which is infinite, semialgebraically connected, noncommutative, of least possible dimension. Since $G$ is closed and bounded and $H_{1}, H_{2}$ are closed in $G$, they are also closed and bounded. By Lemma 2.27, $B$ is not nilpotent. $B$ has finite center, otherwise, by Fact 2.11, $B / Z(B)$ is again a nonabelian section of smaller dimension than $B$.

Since $B$ is definably connected, $B / Z(B)$ is centerless (2.11), hence by changing $H_{2}$, we may assume that $B$ is centerless. Given $a \in B$, we have already seen $C_{B}(a)$ is infinite, properly contained in $B$ ( $B$ is centerless), and therefore by the minimality of $B, C_{B}(a)^{\circ}$ is abelian. We can now apply Lemma 2.26]

2.3.2. The $R$-isotropic case. Again, we assume here that $R$ is a real closed field, $G$ a $\mathbb{G}$-definably-simple linear group definable in the field $R . \bar{G}$ is still the Zariski closure of $G$ inside $K$, the algebraic closure of $R ; H$ the algebraic connected component of $\bar{G}$.

We assume now that $H$ is $R$-isotropic. It is easy to see that $H$ has no algebraic subgroups defined over $R$ which are infinite and normal, but we only use the fact that $H$ is reductive and has finite center. Our first goal is to find a connected, solvable algebraic subgroup $B$ of $H$, with finite center (in particular, nonnilpotent), which is defined over $R$. This is well-known, but to actually extract it from the literature requires a few words. All the references below come from [2]. We thank Gopal Prasad for pointing those out to us.

An $R$-parabolic subgroup of $H$ is an $R$-subgroup $P$ such that $H / P$ is complete. Since $H$ is $R$-isotropic, it contains a nontrivial minimal $R$-parabolic subgroup $P$ (see 20.6 (ii)), which itself contains some maximal $R$-split torus $T$. If $L=C_{H}(T)$, then $L$ is a Levi subgroup of $P(20.6)$, therefore $P$ can be written as a semidirect product $L \cdot R_{u} P$ where $R_{u} P$ is the unipotent radical of $P$. L and $R_{u} P$ have trivial intersection and both of these groups are defined over $R$. Let $B=T \cdot R_{u} P$.

Claim 2.29. $B$ is connected, solvable and has finite center. In particular, $B$ is not nilpotent.

Proof. As $T$ and $R_{u} P$ are connected, so is $B$. Since $R_{u} P$ contains only unipotent elements, it is a nilpotent group. $T$ is commutative and acts by conjugation on $R_{u} P$ so $B$ is solvable.

We show that $Z(B) \subseteq Z(H)$. Suppose that $x \in Z(B)$. Then $x$ centralizes $T$ so $x \in L \cap B$, which is equal to $T$. But then $x$ centralizes $L$ and as $x$ centralizes $R_{u} P$ it also centralizes $L \cdot R_{u} P=P$. Since $H / P$ is complete, $C_{H}(P)=Z(H)$, (see 11.4), hence $x \in Z(H)$.

$B=B(K)$ is then an $R$-group which is solvable and has finite center, therefore $B(R)$ is solvable of finite center.

Since $H(R) \cap G$ has finite index in both $H(R)$ and $G$ it follows that $D=B(R) \cap G$ is still solvable. We claim that $D$ is not nilpotent-by-finite. If it were, then $D^{0}$, the semialgebraic connected component of $D$, is nilpotent. But then the Zariski closure 
of $D^{0}$ is nilpotent as well, and has the same algebraic dimension as $B$, contradicting the fact that $B$ is not nilpotent-by-finite.

Using centers of centralizers one obtains a $\mathbb{G}$-definable solvable subgroup containing $D$, which therefore is not nilpotent and moreover not nilpotent-by-finite (since $D$ is not). We thus found a $\mathbb{G}$-definable solvable group which is not nilpotent-byfinite.

By Theorem 2.12 a field is interpretable in $\mathbb{G}$. We have thus completed the proof that a field is interpretable in a semialgebraic, definably simple, infinite group.

Remark 2.30. In the last argument, in order to obtain a solvable, nonnilpotent group, we only used the fact that the field $R$ was of characteristic zero.

\section{The BI-INTERPRETABILITy of $G$ AND $K$}

So far we have shown how one can interpret a field $K$ in the group structure $\mathbb{G}=\langle G, \cdot\rangle$, when $G$ is an $R$-semialgebraic, definably simple group in some real closed field $R$. By [12], $\operatorname{char}(K)=0$ and $K$ is either algebraically closed or real closed. Our goal now is to show that $K$ can be replaced, if needed, by another $\mathbb{G}$-interpretable field $k$ such that $\langle G, \cdot\rangle$ is bi-interpretable with $k$.

Our first claim is that we may assume that every $\mathbb{G}$-definable subset of $K^{n}$ is definable in $\langle K,+, \cdot\rangle$.

Assume that $K$ is a real closed field. $G$ and hence $K$ are semialgebraically definable in $R$ and therefore, by [12, there is a semialgebraic isomorphism between $K$ and $R$. It follows that every $R$-definable subset of $K^{n}$ is already definable in $\langle K,+, \cdot\rangle$.

Assume now that $K$ is algebraically closed. By [12, it is definably isomorphic in $R$ to $R(\sqrt{-1})$. Thus $K=k(\sqrt{-1})$, for a real closed field $k$ definable in the field $R$. Since the isomorphism between $R$ and $k$ is definable in $R$ every $R$-definable subset of $k^{n}$ is definable in the field $k$. If $A \subseteq K^{n}$ is a definable set in $\mathbb{G}$, possibly over parameters outside of $K$, then it is semialgebraic in the sense of $k$. Consider the expansion $\mathcal{K}_{A}$ of $K$ obtained by adding a predicate for $A$.

We can now apply a theorem of Marker (see [10]) to $\mathcal{K}_{A}$. The theorem states

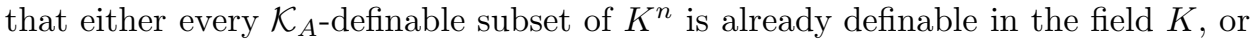
$k$ itself is definable in $\mathcal{K}_{A}$. (Marker proves it for semialgebraic expansions of $\mathbb{C}$ but exactly the same proof works when $\mathbb{R}$ is replaced by an arbitrary real closed field.)

If there is a $\mathbb{G}$-definable set $A$ such that $k$ is definable in $\mathcal{K}_{A}$, then we replace $K$ with $k$, and continue as above. If not, then every $\mathbb{G}$-definable subset of $K^{n}$ is definable in $K$.

As shown so far, we may assume in both cases that every $\mathbb{G}$-definable subset of $K^{n}, n \in \mathbb{N}$, is definable in the field structure of $K$. We claim that the same holds for $K$ in any elementary extension of our structure. In the case that $K$ is real closed it is clear since $K$ and $R$ are still semialgebraically isomorphic. When $K$ is algebraically closed, if the above failed in some elementary extension, then by Marker's result a real closed field is definable in $\mathcal{K}_{A}$ for a $\mathbb{G}$-definable set $A$. By quantifying over the parameters defining this field and $A$ the same holds in our ground model contradicting our assumption.

We may therefore use the compactness theorem to conclude that any $\mathbb{G}$-definable family of subsets of $K^{n}$ is definable in the field $K$. More precisely, for every formula $\phi(x, y)$ in the group language there exists a formula $\psi(x, z)$ in the field language, 
possibly over parameters from $K$, such that

$$
\left\{\phi\left(K^{n}, a\right): a \in G^{n}\right\}=\left\{\psi\left(K^{n}, b\right): b \in K^{r}\right\} .
$$

We now prove a general lemma on bi-interpretability.

Lemma 3.1. Let $\mathcal{N}_{1}, \mathcal{N}_{2}$ be arbitrary structures. Assume that $\mathcal{N}_{1}$ is interpretable in $\mathcal{N}_{2}$ via the identity map, and that every $\mathcal{N}_{2}$-definable subset of $N_{1}^{k}, k \in \mathbb{N}$, is $\mathcal{N}_{1}$-definable. Assume also that there is an $\mathcal{N}_{2}$-definable surjection from $N_{1}^{r}$ onto $N_{2}$ for some $r$.

Then $\mathcal{N}_{1}$ and $\mathcal{N}_{2}$ are bi-interpretable.

Proof. Assume that $\sigma: N_{1}^{r} \rightarrow N_{2}$ is such an $\mathcal{N}_{2}$-definable surjection. Then there is an $\mathcal{N}_{2}$-definable equivalence relation $E$ and an $\mathcal{N}_{2}$-definable bijection $f: N_{2} \rightarrow$ $N_{1}^{r} / E$. $f$ transfers the atomic relations of $\mathcal{N}_{2}$ to some $\mathcal{N}_{2}$-definable relations on cartesian powers of $N_{1}^{r} / E$. But by our assumptions every such relation is $\mathcal{N}_{1^{-}}$ definable, therefore the map $f$ is an interpretation of $\mathcal{N}_{2}$ in $\mathcal{N}_{1}$. Then $i d \circ f$ is an interpretation of $\mathcal{N}_{2}$ in itself, which is $\mathcal{N}_{2}$-definable, and $f \circ i d$ is an interpretation of $\mathcal{N}_{1}$ in itself, which is $\mathcal{N}_{2}$-definable and therefore, by the above, also $\mathcal{N}_{1}$-definable.

In order to prove the bi-interpretability of $\mathbb{G}$ and $K$ it is thus left to prove the existence of a $\mathbb{G}$-definable surjection from $K^{r}$ onto $G$ for some $r$. To do so we develop some general model theoretic machinery for o-minimal structures and more generally, for geometric structures.

\subsection{Geometric structures and $\mathcal{A}$-minimal sets.}

Definition 3.2. A first order structure $\mathcal{M}$ is called a geometric structure if algebraic closure has the Exchange property and $\mathcal{M}$ satisfies the Uniform Bound property: Given a formula $\phi(x, y)$ there is $k$ such that for all $a \in M^{k}$ either $\phi(x, a)$ has infinitely many solutions or no more than $k$ solutions.

The notion of a geometric structure was introduced in [9]. o-minimal and strongly minimal structures are geometric structures. So are p-adically closed fields, perfect PAC fields and real closed rings.

Throughout this section $\mathcal{M}$ is a fixed geometric structure.

We refer the reader to Section 2 in [9] for more information on geometric structures. Here we will mainly use the existence of well behaved notions of dimensions for definable sets and for tuples. Since $\operatorname{acl}(-)$ satisfies the Exchange principle, it generates a pre-geometry with $\operatorname{acl}(-)$ as the closure operation, acting on subsets of $M$. Given $X \subseteq M$ and $A \subseteq M$, the dimension of $X$ over $A$, $\operatorname{dim}(X / A)$, is defined to be the size of any maximal acl-independent-over- $A$ subset of $X$. If $\mathcal{M}$ is $\omega_{1}$-saturated and $D \subseteq M^{n}$ is definable over some finite set $A$, then one can define the dimension of $D$ as $\max \{\operatorname{dim}(\bar{d} / A): \bar{d} \in D\}$. This definition does not depend on the set $A$ that we choose. A point $\bar{d} \in D^{n}$ is called generic in $D$ over $A$ if $\operatorname{dim}(\bar{d} / A)=\operatorname{dim} D$. We have the following dimension formula for $a, b$ some tuples in $\mathcal{M}$ and $A \subseteq M$.

$$
\operatorname{dim}(a, b / A)=\operatorname{dim}(a / b A)+\operatorname{dim}(b / A) .
$$

As pointed out in [9], the notion of dimension for definable sets is itself definable in $\mathcal{M}$. Namely, given a definable set $Y \subseteq M^{k+n}$ and a natural number $r$, the collection of $a \in M^{k}$ such that $\operatorname{dim} Y_{a}=r$ is a definable set in $\mathcal{M}$. For an equivalent definition of dimension where $\mathcal{M}$ needs no saturation assumptions, see [9]. 
Assume now that $\mathcal{A}$ is a structure which is definable in $\mathcal{M}$. Namely, the underlying set $A$ is a definable subset of $M^{k}$ for some $k$ and the atomic relations in $\mathcal{A}$ are $\mathcal{M}$-definable sets. For example, $\mathcal{A}$ could be a group which is definable in a structure $\mathcal{M}$.

Definition 3.3. A definable set $X \subseteq A^{k}$ is called $\mathcal{A}$-minimal if it is infinite and every $\mathcal{A}$-definable subset of $X$ is either finite or has the same dimension as $X$, in the sense of $\mathcal{M}$.

Suppose that $X$ is a definable set in the structure $\mathcal{A}$ over parameter set $B$. We denote by $\mathcal{X}$ the structure that $\mathcal{A}$ induces on $X$. Its universe is $X$ and its 0 -definable sets are the intersection of all definable sets over $B$ in $\mathcal{A}$ with $X^{k}$, for different $k$.

Lemma 3.4. If $\mathcal{A}$ is definable in $\mathcal{M}$ and $X$ is an $\mathcal{A}$-minimal set definable in $\mathcal{A}$, then $\mathcal{X}$ is a geometric structure.

Proof. Since $\mathcal{M}$ is a geometric structure, it is easy to see that for every $\mathcal{A}$-definable family of subsets of $X^{n}$ there is a natural number $k$ such that every set in the family is either infinite or has less than $k$ elements. It follows that $\mathcal{X}$ has the Uniform Bound property.

To see that algebraic closure in $\mathcal{X}$ satisfies the Exchange property, let $a \in X$ be in the $\mathcal{A}$-algebraic closure of $b \in X$ but not in the $\mathcal{A}$-algebraic closure of $\emptyset$. It is sufficient to show that $b$ is in the $\mathcal{A}$-algebraic closure of $a$. Let $Y \subseteq X^{2}$ be a 0 -definable set in $\mathcal{X}$ such that $(a, b) \in Y$ and there are finitely many $x \in X$ for which $(x, b) \in Y$. If there are finitely many $y \in X$ such that $(a, y) \in Y$, then we are done. So we assume, towards contradiction, that there are infinitely many such $y$ 's.

Let $\pi_{1}, \pi_{2}$ be the projection maps on the first and second coordinates, respectively. $\pi_{1}(Y), \pi_{2}(Y)$ are infinite, since otherwise $a$ is algebraic over the empty set, therefore by assumptions on $X$ these sets have the same $\mathcal{M}$-dimension as $X$. By the Uniform Bound property, we may assume that for every $x \in \pi_{1}(Y)$ there are infinitely many $y \in X$ such that $(x, y) \in Y$. By definition of dimension in geometric structures, the $\mathcal{M}$-dimension of $Y$ is strictly bigger than that of $X$.

On the other hand, by our assumption on $(a, b)$, we may assume that for every $y \in \pi_{2}(Y)$ there are finitely many $x \in X$ such that $(x, y) \in Y$. It follows that $\operatorname{dim} Y=\operatorname{dim} X$, contradiction.

We thus have two geometric structures present, $\mathcal{M}$ and $\mathcal{X}$. We use $\operatorname{dim}$ and $\operatorname{dim}_{\mathcal{X}}$ to denote the dimension of sets or tuples with respect to $\mathcal{M}$ and $\mathcal{X}$, respectively.

Lemma 3.5. For $\mathcal{A}$ and $\mathcal{X}$ as above, if $Y \subseteq X^{n}$ is a $\mathcal{X}$-definable set, then $\operatorname{dim} Y=$ $\operatorname{dim} X \cdot \operatorname{dim}_{\mathcal{X}} Y$.

Proof. We use induction on $n$.

For $n=1$, the statement follows from the minimality of $X$. We assume it holds for $n$ and let $Y \subseteq X^{n+1}$ be definable in $\mathcal{X}$. Let $\pi: X^{n+1} \rightarrow X^{n}$ be the projection map and let

$$
\begin{gathered}
Y_{1}=\left\{a \in \pi(Y): Y_{a} \text { is finite }\right\}, \\
Y_{2}=\left\{a \in \pi(Y): Y_{a} \text { is infinite }\right\}=\left\{a \in \pi(Y): \operatorname{dim} Y_{a}=\operatorname{dim} X\right\},
\end{gathered}
$$

(the last equality follows from the minimality of $X$ ). 
For $i=1,2$, let $Y_{i}^{\prime}=\pi^{-1}\left(Y_{i}\right) \cap Y$. Each $Y_{i}$ and $Y_{i}^{\prime}$ is definable in $\mathcal{X}$, and by properties of geometric structures we have,

$$
\operatorname{dim} Y_{1}^{\prime}=\operatorname{dim} Y_{1} ; \operatorname{dim}_{\mathcal{X}} Y_{1}^{\prime}=\operatorname{dim}_{\mathcal{X}} Y_{1},
$$

and

$$
\operatorname{dim} Y_{2}^{\prime}=\operatorname{dim} Y_{2}+\operatorname{dim} X ; \operatorname{dim}_{\mathcal{X}} Y_{2}^{\prime}=\operatorname{dim}_{\mathcal{X}} Y_{2}+1
$$

By induction, $\operatorname{dim} Y_{i}=\operatorname{dim}_{\mathcal{X}} Y_{i} \cdot \operatorname{dim} X, i=1,2$.

Since the dimension of $Y$, in $\mathcal{M}$ and in $\mathcal{X}$, is the maximum of dimensions of $Y_{1}^{\prime}$ and $Y_{2}^{\prime}$, we have

$$
\operatorname{dim} Y=\operatorname{dim}_{\mathcal{X}} Y \cdot \operatorname{dim} X
$$

The structure $\mathcal{A}$ which we are going to consider in this paper is not necessarily definable in $\mathcal{M}$ but instead could be interpretable there. Similarly, we will consider sets $X$ which are obtained as quotients of $\mathcal{A}$-definable sets by $\mathcal{A}$-definable equivalence relations. We therefore need to develop some general theory for such quotients in geometric structures. We will assume that the reader is familiar with the notion of $\mathcal{M}^{e q}$.

As is shown in [16], the following property holds in every o-minimal structure.

Property E. For every definable subset $U$ of $M^{n}$ and a definable equivalence relation $E$ on $U$, if $E$ has infinitely many classes, each of dimension at least $k$, then $\operatorname{dim} U>k$.

We assume now that $\mathcal{M}$ is a geometric structure satisfying Property $\boldsymbol{E}$.

In $\mathcal{M}$, one can define the dimension of definable quotients in exactly the same way as in [13] for o-minimal structures. Given $U \subseteq M^{r}$ and $a \in U$ we will use $[a]$ to denote the equivalence class of $a$, which is clearly a definable subset of $U$, and let $\dot{a}$ denote the element in $\mathcal{M}^{e q}$ which corresponds to $[a]$. Given $A \subseteq M^{e q}$, we can then define

$$
\operatorname{dim} \dot{a} / A=\operatorname{dim} a^{\prime} / A-\operatorname{dim}[a],
$$

where $a^{\prime}$ is generic in the set $[a]$ over $a$. If we assume that $\mathcal{M}$ is $\omega_{1}$-saturated, then an equivalent definition to the one in 13 is

$$
\operatorname{dim} \frac{U}{E}=\max \{\operatorname{dim} \dot{a}: a \in U\}
$$

Using Property E, it is shown in [13] that all expected properties hold for this notion of dimension. The notion of dimension is a definable notion in $\mathcal{M}^{e q}$ in the same sense it was in $\mathcal{M}$. From the dimension formula in $\mathcal{M}$ follows the dimension formula in $\mathcal{M}^{e q}$. Assume that $\dot{a}, \dot{b} \in M^{e q}$, given via the equivalence relations $E_{1}, E_{2}$ respectively. Then $(\dot{a}, \dot{b})$ is again an element of $M^{e q}$ via $E_{1} \times E_{2}$. If $A \subseteq M^{e q}$, we then have

$$
\operatorname{dim}(\dot{a}, \dot{b}) / A=\operatorname{dim} \dot{a} / \dot{b} A+\operatorname{dim} \dot{b} / A
$$

Assume now that $\mathcal{A}$ is a structure which is interpretable in $\mathcal{M}$, via the identity map. That is, the universe of $A$ is a definable quotient in $\mathcal{M}$ and its atomic relations are the traces of definable sets in $\mathcal{M}$ on cartesian powers of $A$. Assume that $X$ is a definable quotient in $\mathcal{A}$. We say that $X$ is $\mathcal{A}$-minimal if it is infinite and every $\mathcal{A}$-definable infinite subset of $X$ (which is a definable subset of $\mathcal{M}^{e q}$ ) has the same dimension as $X . \mathcal{X}$ still denotes the structure which $\mathcal{A}$ induces on $X$ as before. 
Lemma 3.6. If $\mathcal{A}$ is interpretable in $\mathcal{M}$ and $X$ is $\mathcal{A}$-minimal, obtained as a definable quotient in $\mathcal{A}$, then $\mathcal{X}$ is a geometric structure.

Proof. The Uniform Bound property follows from the definability of dimension in $\mathcal{M}^{e q}$. The proof of the Exchange property is almost identical to the proof of Lemma 3.4 and we omit it.

The conclusion of Lemma 3.5 still holds for $\mathcal{A}$ and $\mathcal{X}$ as above, with identical proof. Applying this lemma to the dimension of definable sets in $\mathcal{X}$ and to the dimension of equivalence classes under definable equivalence relations in $\mathcal{X}$ we can conclude that $\mathcal{X}$ itself has property $\mathbf{E}$. We can now phrase a more general statement.

Lemma 3.7. Assume that $\mathcal{A}$ and $X$ are as above. If $Y$ is an $\mathcal{X}$-definable subset of $X^{e q}$, then $\operatorname{dim} Y=\operatorname{dim} X \cdot \operatorname{dim}_{\mathcal{X}} Y$.

We end this section with a technical lemma which we will use later on. It can be seen as a weak version of elimination of imaginaries.

Lemma 3.8. Assume that $E$ is a definable equivalence relation on $M^{n}$. Then, there is a definable $S \subseteq M^{n}$ such that for every $x \in S,[x] \cap S$ is finite ([x] is the equivalence class of $x)$, and $\operatorname{dim} S=\operatorname{dim}(S / E)=\operatorname{dim}\left(M^{n} / E\right)$.

Proof. Without loss of generality, all sets are definable over $\emptyset$. By the definition of dimension in $\mathcal{M}^{e q}$, there is a definable set $U \subseteq M^{n}$, such that the dimension of $[x]$ is fixed as $x$ varies over $U$, call it $l$, and $\operatorname{dim} M^{n} / E=\operatorname{dim} U-l$. Furthermore, if $a \in U$, then $[a] \subseteq U$. We fix $b$ a generic element in $U$ and take $a=\left(a_{1}, \ldots, a_{n}\right)$ generic in $[b]$ over $b$, i.e. $\operatorname{dim}(a / b)=l$. By reordering the coordinates we may assume that $a_{1}, \ldots, a_{l}$ are acl-independent over $b$ and that there are finitely many $x^{\prime} \in M^{n-l}$ such that $\left(a_{1}, \ldots, a_{l}, x^{\prime}\right)$ is $E$-equivalent to $b$ (in particular, it is in $U$ ). Using dimension equalities, $a$ is itself generic in $U$ over $\emptyset$, hence $\operatorname{dim}\left(a / a_{1}, \ldots, a_{l}\right)=k-l$, where $k=\operatorname{dim} U$.

Let

$$
Y=\left\{\left(a_{1}, \ldots, a_{l}, x^{\prime}\right) \in U: x^{\prime} \in M^{n-l}\right\} .
$$

Since $a$ is generic in $Y$ over $a_{1}, \ldots, a_{l}$ we have $\operatorname{dim} Y=k-l$. Let

$$
S=\left\{\left(a_{1}, \ldots, a_{l}, x^{\prime}\right) \in Y:\left[\left(a_{1}, \ldots, a_{l}, x^{\prime}\right)\right] \cap Y \text { is finite }\right\} .
$$

Since $a \in S \subseteq Y$ and $S$ is definable over $a_{1}, \ldots, a_{l}, \operatorname{dim} S=k-l=\operatorname{dim}\left(M^{n} / E\right)$, and by definition, if $x \in S$, then $[x] \cap S$ is finite. It follows that $\operatorname{dim} S=\operatorname{dim}(S / E)$.

3.2. Definable infinitesimal subgroups and their Lie algebras. We assume here that $\mathcal{M}$ is an o-minimal expansion of a real closed field.

We now return to the notion of a Lie algebra which is associated to certain subgroups of a definable group $G$ in an o-minimal expansion of a real closed field. Most of this treatment was carried out in [14] and we refer the reader to it for definitions and basic results. The only difference here is that we will work not with definable subgroups of $G$ but with $\bigwedge$-definable "infinitesimal subgroups" of $G$.

Definition 3.9. Assume that $G$ is a definable group in $\mathcal{M}, Y \subseteq G$ a definable subset. If $H=Y \cap \mathcal{V}_{e}$ is a subgroup of $G$, then $H$ is called a $\bigwedge$-definable subgroup.

If $H$ is a $\bigwedge$-definable subgroup of $G$, then we define the dimension of $H$ to be its dimension as a partial type. Namely, $\operatorname{dim} H$ is the minimum of $\operatorname{dim}(Y \cap V)$, for $V$ an $M$-definable open neighborhood of $e$. 
If $G$ is a definable group in an o-minimal expansion of a real closed field $R$, then for every $p \geq 0$ it carries a definable $C^{p}$-manifold making the group operations $C^{p}$-maps.

Lemma 3.10. Let $H$ be a $\bigwedge$-definable subgroup of $G$ of dimension $k$. Then there is a definable set $V \subseteq H$ containing $e, V$ open in the topology induced on $H$ by $G$, and a definable function $\tau$ from $V$ into $R^{k}$ such that $\langle V, \tau, k\rangle$ is a definable $C^{p}$ chart on $V$. $V$ with this single definable chart is a definable submanifold of $G$. The maps $h \mapsto h^{-1}$ and $\left(h_{1}, h_{2}\right) \mapsto h_{1} h_{2}$ are partial $C^{p}$-maps (in this definable manifold structure) from $V$ into $V$, and from $V \times V$ into $V$, respectively.

Proof. Let $\langle U, \phi, n\rangle$ be a definable $C^{p}$ chart on $G$ at $e$. We will assume that $\phi$ is the identity. We may assume that $U^{-1}=U$, therefore the inverse map is a $C^{p}$-map from $U$ into $U$ and group multiplication is a partial $C^{p}$-map from $U \times U$ into $U$.

Let $H=Y \cap \mathcal{V}_{e}$ for a definable set $Y$. We assume that $Y$ is $\emptyset$-definable of dimension $k$ and choose $h \in U \cap H$ which is generic in $Y$ over $M$. There is then a $k$-cell $C \subseteq H \cap U$ containing $h$ and a definable bijection $f$ between $C$ and a definable open $D \subseteq R^{k}$. We can choose $C$ and $f$ so that $h$ is generic in $C$ over all mentioned parameters, therefore we may assume that $f^{-1}$ is a $C^{p}$-map from $D$ into $U$ and furthermore $d_{x}\left(f^{-1}\right)$ is injective for every $x \in D$. The definable chart $\langle C, f\rangle$ makes $C$ into a definable manifold which is a definable submanifold of $G$.

Now consider $V=h^{-1} \cdot C$. Since $C$ is an open subset of $H$ in the subset topology so is $V$. The map $g \mapsto f(h g)$ is a definable bijection between $V$ and $D$. The map $g \mapsto h^{-1} g$ is a $C^{p}$-diffeomorphism from $G$ to itself therefore the map $x \mapsto h^{-1} f^{-1}(x)$ still has injective differential at every $x \in D$. Thus $V$ has a definable $C^{p}$-submanifold structure. It is easy to verify that the group operations are $C^{p}$ on $V$ when defined.

As seen in 14, given a definable $C^{p}$-manifold $X$, one can define at every $x \in X$ the tangent space $T_{x}(X)$. If $G$ is a definable group, then $T_{e}(G)$ carries a Lie algebra structure, denoted by $\mathcal{L}(G)$. To every definable subgroup $G_{1}$ of $G$ corresponds a Lie subalgebra $\mathcal{L}\left(G_{1}\right) \subseteq \mathcal{L}(G)$. If $G_{1}$ is normal in $G$, then $\mathcal{L}\left(G_{1}\right)$ is an ideal in $\mathcal{L}(G)$. We proved there (Corollary 4.3) the following (note that unlike [14], when we use here "definably simple" we refer to definability in the sense of $\mathbb{G}$ ).

Theorem 3.11. Let $G$ be a definably simple group which is definable in $\mathcal{M}$. Then $\mathcal{L}(G)$ is a simple Lie algebra.

We now can prove the main corollary which we will use here.

Theorem 3.12. Let $G$ be a definably simple group which is definable in $\mathcal{M}$. If $H$ is a \-definable normal subgroup of $\mathcal{V}_{e}$, then either $H=\{e\}$ or $H=\mathcal{V}_{e}$

Proof. First, by the results in [14], we may assume that $G$ is semialgebraic over a real closed field $R$. Assume that $H$ is such a normal subgroup of $\mathcal{V}_{e}$. By Lemma 3.10 there is a definable open neighborhood of $e$ in $H$, call it $V$, which carries a definable $C^{p}$-submanifold structure, making the group operations, as maps into $V$, $C^{p}$-maps when defined.

We can view $T_{e}(V)$ as a subspace of $T_{e}(G)$. Consider the subgroup

$$
G_{1}=\left\{g \in G: \operatorname{Ad}(g)\left(T_{e}(V)\right) \subseteq T_{e}(V)\right\} .
$$

Note that for every $g \in \mathcal{V}_{e}$, since $g$ leaves $H$ invariant by conjugation, its differential Ad $(g)$ leaves $T_{e}(V)$ invariant inside $T_{e}(G)$. Therefore, $\operatorname{dim} G_{1}=G$ and hence the 
Lie algebra of $G_{1}$ equals $\mathcal{L}(G)$. By Claim 2.30(2) in [14, $T_{e}(V)$ is an ideal inside $\mathcal{L}(G)$. But, by 3.11, $\mathcal{L}(G)$ is simple, therefore, either $\operatorname{dim} H=0$ or $\operatorname{dim} H=\operatorname{dim} G$. In the latter case, $H$ contains an open set around 0 so, if $H=Y \cap \mathcal{V}_{e}$ for a definable $Y$, then $Y$ contains an open set around 0 and thus $\mathcal{V}_{e} \subseteq Y$.

Corollary 3.13. Assume that $G$ is a definably simple group which is definable in $\mathcal{M}$. Assume that $Y \subseteq G$ is an $\mathcal{M}$-definable infinite set containing e. Then there are $g_{1}, \ldots, g_{m} \in G$ such that the set $\left(Y^{ \pm 1}\right)^{g_{1}} \cdots\left(Y^{ \pm 1}\right)^{g_{m}}$ contains an open neighborhood of $e$.

Proof. By Corollary 2.5, there are $g_{1}, \ldots, g_{m} \in G$ such that if we let $X=\left(Y^{ \pm 1}\right)^{g_{1}}$ $\cdots\left(Y^{ \pm 1}\right)^{g_{m}}$, then $X \cap \mathcal{V}_{e}$ is normal in $\mathcal{V}_{e}$. By Corollary 3.12, $X$ contains $\mathcal{V}_{e}$.

Remark 3.14. For the rest of the proof of the bi-interpretability result, we use the fact that $G$ is definable in a geometric structure with property $\mathbf{E}$, together with Corollary 3.13 No other property of o-minimal structures is assumed. This raises the question whether Corollary 4.7 holds when $\mathcal{M}$ is assumed to be a geometric structure.

The following proposition and subsequent discussion will not be used in the rest of the paper. A similar theorem, due to Riehm, is proved in [18] (see Theorem 3.3 there) for locally compact fields. It is noted there that the theorem is true in every field in which the inverse function theorem holds, as indeed the case is here for definable functions. A strong version of this theorem is proved in [17] for the reals and p-adics (see 3.4 and 3.5 there).

Proposition 3.15. Assume that $G$ is a definably simple group which is definable in $\mathcal{M}$. Then every normal subgroup of $G$ (not necessarily definable) is open.

Proof. As we already pointed out before, $G$ is $\mathbb{G}$-definably connected. If $H$ is a normal subgroup of $G$ and $a \in H$, then we consider its conjugacy class $a^{G}$ which is definable in $\mathbb{G}$. Since $\left|a^{G}\right|=\left|G / C_{G}(a)\right|$ the set $Y=a^{-1} a^{G}$ is an infinite $\mathbb{G}$-definable subset of $H$ containing $e$. By Corollary 3.13 , there are finitely many conjugates of $Y$ (or of $Y^{-1}$ ) such that their product $X$, which is contained in $H$, has the same dimension as $G$. But then $X$ contains an open set and by standard arguments, $H$ must be open.

When we work over an arbitrary real closed field, then a definably simple group might have open normal subgroups which are nontrivial. For example, the group of infinitesimals in $\mathrm{SO}(3, R)$ when $R$ is nonarchimedean is a normal subgroup. However, when $R$ is the field of real numbers this is impossible, as is shown in [17, therefore a definably simple group over the reals is abstractly simple.

\section{INTERNALITY IN DEFINABLY SIMPLE GROUPS}

In this section $\mathcal{M}$ is an $\omega_{1}$-saturated o-minimal structure.

We take $G$ to be a definable group in $\mathcal{M}$. Our interest here is with the group structure $\langle G, \cdot\rangle$, or more generally, with some expansion of $G$ by some $\mathcal{M}$-definable sets which we denote $\mathbb{G}$.

Definition 4.1. For $D$ and $V$ definable sets in $\mathbb{G}^{e q}$ we say that $V$ is $D$-internal in $\mathbb{G}$ if there is a finite set $A \subseteq G$ such that $V \subseteq \operatorname{dcl}(D \cup A)$, where dcl is taken in the structure $\mathbb{G}$. 
The following observation indicates why the notion of internality is of interest to us.

Lemma 4.2. If $X, Y$ are definable sets in $\mathbb{G}^{e q}$ such that $X$ is $Y$-internal, then there is a definable map from $Y^{r}$ onto $X$ for some $r$.

Proof. By the saturation of $\mathcal{M}$, there are finitely many formulas in the language of $\mathbb{G}$, such that every element in $X$ is in the definable closure of some tuple from $Y$, via one of these formulas. It is easy to see that we can now find a $\mathbb{G}$-definable map from $Y^{r}$ onto $X$, for some $r$.

We say that a definable $U \subseteq G$ is of full dimension if $\operatorname{dim} U=\operatorname{dim} G$. Our first goal is to prove

Theorem 4.3. Assume that $G$ is an infinite, definably simple group and $\mathbb{G}$ an expansion of $G$ by $\mathcal{M}$-definable sets. If $E$ is a $\mathbb{G}$-definable equivalence relation on $G^{n}$ such that $D=G^{n} / E$ is infinite, then there is a $\mathbb{G}$-definable $V \subseteq G$ of full dimension such that $V$ is D-internal in $\mathbb{G}$.

Unless we specify otherwise, we use the word definable below for sets definable in $\mathbb{G}$. The following lemma will be used in the subsequent arguments.

Lemma 4.4. Let $Y$ be a definable $\mathbb{G}$-minimal subset of $G$. Then there is a definable $U \subseteq G$ of full dimension such that $U$ is $Y$-internal in $\mathbb{G}$. Moreover, there is a definable finite-to-one map from an infinite definable $S \subseteq Y^{k}$ onto $U$.

Proof. By replacing $Y$ with a translate of it we may assume that it contains $e$. By Corollary 3.13, there are finitely many conjugates of $Y$ and $Y^{-1}$ whose groupproduct, which we call $U$ is of full dimension. It follows that $U$ is $Y$-internal in $\mathbb{G}$. By Lemma 4.2 there is a definable function $\sigma$ from $Y^{k}$ onto $U$.

If we let $E_{1}$ be the natural equivalence relation on $Y^{k}$ which is induced by $\sigma$, then $\operatorname{dim} Y^{k} / E_{1}=\operatorname{dim} U$. By Lemma 3.8 (applied to the geometric structure which $\mathbb{G}$ induces on $Y$ ), there is a definable $S \subseteq Y^{k}$ such that $\operatorname{dim} S=\operatorname{dim} Y / E_{1}=\operatorname{dim} U$ and such that the restriction of $\sigma$ to $S$ is finite-to-one. In particular, $\operatorname{dim}(\sigma(S))=$ $\operatorname{dim} U$. We now can replace $U$ with $\sigma(S)$.

As a first step towards Theorem 4.3, we prove the following lemma.

Lemma 4.5. Let $G, E$ and $D$ be as in Theorem 4.3. Then there is a definable $U \subseteq G$ of full dimension, and a definable equivalence relation $\sim$ on $U$ all of whose classes are finite, such that $U / \sim$ is D-internal in $\mathbb{G}$.

Proof. Let $X$ be a $\mathbb{G}$-minimal subset of $G$. By Lemma 4.4 there is a definable $V \subseteq G$ of full dimension such that $V$ is $X$-internal. By the definition of dimension of quotients, we can find finitely many translates $V_{1}, \ldots, V_{k}$ of $V$, (each $V_{i}$ of the form $g_{i} \cdot V$ for some $\left.g_{i} \in G\right)$ such that $\operatorname{dim}\left(V_{1} \times \cdots \times V_{n} / E\right)=\operatorname{dim}\left(G^{n} / E\right)=\operatorname{dim} D$. In particular, there is an infinite set $D_{1} \subseteq D$ which is $V$-internal in $\mathbb{G}$, and therefore $D_{1}$ is $X$-internal. As in the second part of the proof of Lemma 4.4 there is a definable finite-to-one map $\sigma_{1}$ from a definable $X_{1} \subseteq X^{k}$ onto an infinite subset of $D$.

We now take $Y \subseteq X_{1}$ to be a $\mathbb{G}$-minimal set. By Lemma 4.4, there is a definable set $S \subseteq Y^{r}$ for some $r$, a definable $U \subseteq G$ of full dimension and a definable finiteto-one map $\sigma_{2}$ from $S$ onto $U$. Since $S \subseteq X_{1}^{r}$, the map $\sigma_{1}$ above restricts to a finite-to-one map, which we call $\sigma_{1}$ again, from $S$ onto an infinite subset $D_{2}$ of $D^{r}$. 
We thus have two definable finite-to-one surjective maps on $S$,

$$
\begin{gathered}
\sigma_{1}: S \rightarrow D_{2} \subseteq D^{r} ; \\
\sigma_{2}: S \rightarrow U \subseteq G .
\end{gathered}
$$

Given $g \in U$, we define the set $D_{g} \subseteq D_{2}$ to be

$$
D_{g}=\left\{\sigma_{1}(a): a \in S \& \sigma_{2}(a)=g\right\}=\sigma_{1}\left(\sigma_{2}^{-1}\{g\}\right) .
$$

We obtain a definable family $\left\{D_{g}: g \in U\right\}$ of finite subsets of $D^{r}$. We let $\sim$ be the equivalence relation on $U$ defined by $g_{1} \sim g_{2}$ if and only if $D_{g_{1}}=D_{g_{2}}$. Since $\sigma_{1}$ and $\sigma_{2}$ are both finite-to-one, all $\sim$-classes are finite. We thus have found a definable equivalence relation $\sim$ on $U$ with finite classes and a definable one-to-one map $\pi$ on $U / \sim$ which assigns to each $\sim$-class $[g]$ the finite set $D_{g} \subseteq D^{r}$. It follows that every $[g]$ is in $\operatorname{dcl}(D)$ and therefore $U / \sim$ is $D$-internal.

We point out that until now we only used the fact that $G$ has no definable infinite subgroups. To finish the proof of Theorem 4.3 we need the following lemma.

Lemma 4.6. Assume that $G$ is a definable, infinite connected group with no finite nontrivial normal subgroups. Assume also that $U \subseteq G$ is a $\mathbb{G}$-definable set of full dimension and $\sim$ is a $\mathbb{G}$-definable equivalence relation on $U$ all of whose classes are finite. Then there exists a $\mathbb{G}$-definable $V \subseteq G$ of full dimension which is $(U / \sim)$ internal.

Proof. We may assume that there is $k$ such that every $\sim$-class has exactly $k$ elements. We then have a definable $k$-to-one map from $U$ into $F=U / \sim$. We now take $m$ to be a minimal natural number for which there is a definable set $V \subseteq G$ of full dimension and a definable $m$-to-one map $\sigma: V \rightarrow F^{l}$, for some $l$. If $m=1$, then the lemma follows and we are done. We thus assume for contradiction that $m>1$.

STEP I. We will replace $V$ by a set of full dimension containing $e$ such that for some finite subgroup $H<G$ of size $m, \sigma\left(g_{1}\right)=\sigma\left(g_{2}\right)$ if and only if $g_{1}$ and $g_{2}$ are in the same $H$-coset.

Let $E$ denote the equivalence relation on $V$ induced by $\sigma$ and let $[g]$ denote the $E$-class of an element $g \in V$.

Claim A. If $g_{0}$ is generic in $V$ over $a \in G$ and $a g_{0} \in V$, then $a\left[g_{0}\right]=\left[a g_{0}\right]$.

Proof. Let $g_{0}$ be generic in $V$ over $a, a g_{0} \in V$ and $a\left[g_{0}\right] \neq\left[a g_{0}\right]$. Then there is a set $W$ of full dimension containing $g_{0}$ such that $a W \subseteq V$ and for every $g \in W$ we have $|a[g] \cap[a g]|=m^{\prime}<m$. We can now define an $m^{\prime}$-to-one map from $W$ into $F^{2}$ by $g \mapsto([g],[a g])$, contradicting our choice of $m$, thus proving Claim A.

We now fix $g_{0}$ generic in $V$. Then the set $\left\{a \in G: a g_{0} \in V\right\}$ has full dimension in $G$. By the last claim, if $a$ in this set is independent from $g_{0}$, then $a\left[g_{0}\right]=\left[a g_{0}\right]$. We denote by $\left\{g_{0}, \ldots, g_{m-1}\right\}$ the set $\left[g_{0}\right]$ and let

$$
H=\left\{g_{i} g_{0}^{-1}: g_{i} \in\left[g_{0}\right]\right\} .
$$

Our goal is to prove that $H$ is a subgroup of $G$.

Claim B. For every $g_{i}, g_{j} \in\left[g_{0}\right]$ there is $g_{k} \in\left[g_{0}\right]$ such that $g_{i} g_{0}^{-1}=g_{k} g_{j}^{-1}$. 
Proof. We take $a$ generic in $G$ over the set $\left[g_{0}\right]$ such that $a g_{0} \in V$. By assumptions $a\left[g_{0}\right] \subseteq V$ and $a\left[g_{0}\right]=\left[a g_{0}\right]$. The element $a_{1}=a g_{i} g_{0}^{-1}$ is also generic over the set $\left[g_{0}\right]$ and $a_{1} g_{0}=a g_{i} \in V$, therefore

$$
a_{1}\left[g_{0}\right]=\left[a_{1} g_{0}\right]=\left[a g_{i}\right]=a\left[g_{i}\right]=a\left[g_{0}\right] .
$$

We can now find $g_{k} \in\left[g_{0}\right]$ such that $a_{1} g_{j}=a g_{k}$, which implies that $g_{i} g_{0}^{-1}=$ $g_{k} g_{j}^{-1}$, concluding the proof of Claim B.

Using Claim B, it is a standard argument to see that $H$ is a group. Let

$$
V_{1}=\left\{a g_{0}: a \in G \&\left[a g_{0}\right]=a\left[g_{0}\right] \subseteq V\right\} .
$$

Then every $E$-class in $V_{1}$ is a coset of $H$ and $V_{1}$ is of full dimension.

If $h_{0}$ is generic in $V_{1}$ over $g_{0}$, then $h_{0}^{-1} V_{1}$ is a definable set of full dimension containing $H$ (and an open set around $e$ ). If we replace $V$ by $h_{0}^{-1} V_{1}$, calling it $V$ again we may assume that the number $m$ is realized as the size of a finite subgroup of $G$ and the map which sends $V$ into $(U / E)^{l}$ induces an injection on $V / H$.

STEP II. We will replace $H$ by a finite normal subgroup of $G$.

We still use $E$ to denote the equivalence relation induced by the cosets of $H$ on $V$. Given $a, g \in G$ we let $g^{a}=a g a^{-1}$.

Claim C. Assume that $g$ is generic in $V$ over $a \in G$ and $g^{a} \in V$, then $[g]^{a}=\left[g^{a}\right]$.

Proof. The proof is exactly the same as that of Claim A.

If $g$ is in $V$, then $[g]$ is a coset of $H$, hence $[g]^{a}$ is a coset of $H^{a}$. If $[g]^{a}=\left[g^{a}\right]$, then $a \in N_{G}(H)$. By Claim C, $\operatorname{dim} N_{G}(H)=\operatorname{dim} G$, therefore $N_{G}(H)=G$, contradicting the assumption that $G$ has no normal finite subgroups.

We have shown that $m=1$, thus completing the proof of the lemma, and with it Theorem 4.3.

As we remarked in the last section, the proof of Theorem 4.3 relies on two assumptions only. The first is that $G$ is definable in a geometric structure with property $\mathbf{E}$, and the second that $G$ satisfies the statement in Corollary 3.13 .

Corollary 4.7. Let $G$ be an infinite, definably simple group. Then $G$ is $X$-internal for every $\mathbb{G}$-definable infinite set $X \subseteq \mathbb{G}^{e q}$. In particular, if $K$ is a field interpretable in $\mathbb{G}$, then $G$ is $K$-internal.

Proof. Since every infinite definable set contains a $\mathbb{G}$-minimal set it is sufficient to show, for the first part, that $G$ is $X$-internal for every $\mathbb{G}$-minimal set $X$.

By what was shown in previous sections, a field $K$ is interpretable in $\mathbb{G}$. As we remarked earlier, we may assume that every $\mathbb{G}$-definable subset of $K$ is definable in the field structure alone. If $K$ is algebraically closed, then it has dimension 2 in $\mathcal{M}$ (see [12]). It is strongly minimal and therefore every $\mathbb{G}$-definable subset of $K$ is either finite or co-finite. In particular, $K$ is $\mathbb{G}$-minimal. If $K$ is real closed, then it is of dimension 1 and therefore $\mathbb{G}$-minimal. Therefore in either case, $K$ is $\mathbb{G}$-minimal.

We claim first that it is sufficient to show that $G$ is $K$-internal. Recall that if $K$ is either algebraically closed or real closed, then it has elimination of imaginaries. Now, if $G$ is $K$-internal and $X$ is any other $\mathbb{G}$-minimal set, then $X$ is $K$-internal. As we have observed several times already, there is a definable infinite $X_{1}$ in $K^{e q}$ which is $X$-internal. By elimination of imaginaries, we may assume that $X_{1}$ is a subset of $K^{n}$ and therefore, by projecting onto $K$ we may assume that $X_{1}$ is a subset of $K$. If $K$ is algebraically closed, then $X_{1}$ is co-finite in $K$ and therefore $K$ 
is $X_{1}$-internal. If $K$ is real closed, then $X_{1}$ is a finite union of intervals in $K$. But every such interval is definably (in $K$ ) bijective to $K$ hence $K$ is $X$-internal, and therefore $G$ is $X$-internal. It is thus left to show that $G$ is $K$-internal.

By Theorem 4.3, there is a definable set $U \subseteq G$ of full dimension which is $K$ internal. It follows that there is a definable bijection between a definable $U_{1} \subseteq U$ of full dimension and $K^{n} / E$, for a definable equivalence relation $E$ and some $n$. We again denote $U_{1}$ by $U$. By elimination of imaginaries, $U$ is definably bijective with a subset of $K^{r}$ for some $r$. Without loss of generality, $U$ is a neighborhood of $e$. By replacing $U$ with $U \cap U^{-1}$ we may also assume that $U^{-1}=U$.

Case $1 . K$ is a real closed field.

We may assume now that $U$, together with the structure induced by $G$ is a definable subset of $K^{r}$. By projecting $U$ on some of the coordinates and cutting it down, if needed, we may assume that it is an open subset of $K^{m}$ for some $m$.

As in 3.2.2 in [14], $U$ together with the induced group structure from $G$ can be equipped with a differentiable structure, with respect to the field $K$. Namely, we may assume that the group operation is differentiable in a neighborhood of $e$. It follows (see [14]) that for every $g \in G$ the map $x \mapsto x^{g}$ is differentiable (with respect to $K)$ at a neighborhood of $e$. The map which sends $g$ into $d_{e}\left(x \mapsto x^{g}\right)$ is then an embedding of $G$ into $\mathrm{GL}(r, K)$. In particular, $G$ is $K$-internal.

Case $2 . K$ is algebraically closed.

Let $m=\operatorname{dim} G=\operatorname{dim} U$ (where the dimension is taken in the sense of $\mathcal{M}$ ). We may assume that $U$ is a subset of $K^{r}$ and hence, by Lemma 3.5. as $\operatorname{dim} K=2$, we have $\operatorname{dim}_{K}(U)=m / 2$. But $K$ is a strongly minimal structure, therefore $\operatorname{dim}_{K}(U)$ equals the Morley Rank of $U$. If we now replace $U$ with a definable subset of the same Morley rank but of multiplicity 1 , then again by Lemma 3.5, this set has dimension $m$ in $\mathcal{M}$. We thus may assume that $U$ has multiplicity 1 . Let

$$
H=\operatorname{Stab}(U)=\left\{a \in G: \operatorname{dim}_{K}(a U \cap U)=m / 2\right\} .
$$

As we pointed out at the beginning of Section 3 , the family $\{a U \cap U: a \in G\}$ is definable inside $K$. Since dimension is definable in $K$, the set $H$ is $\mathbb{G}$-definable. Moreover, if $\operatorname{dim}_{K}(a U \cap U)=m / 2=\operatorname{dim}_{K} U$, then because of the multiplicity of $U$, we have $\operatorname{dim}_{K}(U \backslash a U)<m$. It easily follows that $H$ is a subgroup of $G$.

If we take $a$ in a sufficiently small neighborhood of $e, a \cdot U \cap U$ contains an open neighborhood of $e$, so its $K$-dimension is $m / 2$ and $a \in H$. Thus, $\operatorname{dim}(H)=m=$ $\operatorname{dim} G$ and so $H=G$. It follows that $G=U \cdot U$, so in particular $G \subseteq \operatorname{dcl}(K)$.

Let $K$ be the field interpretable in $\mathbb{G}$, which we assume carries no other structure induced from $\mathbb{G}$ other than that given by the field operations. By Corollary $4.7 \mathbb{G}$ is $K$-internal. By Lemma 4.2 and Lemma $3.1 \mathbb{G}$ and $K$ are bi-interpretable.

We have thus proved the bi-interpretability statements in Theorem 1.1. Before finishing the proof of the theorem we prove a general proposition from which Corollary 1.3 immediately follows.

Proposition 4.8. Let $G$ be a semialgebraic infinite group over a real closed field $R$. If $G$ is bi-interpretable with a real closed field, then the following hold.

1. Every semialgebraic subset of $G^{n}$ is $\mathbb{G}$-definable.

2. Let $H$ be any another semialgebraic group over a real closed field L. Then every abstract group isomorphism $f: G \rightarrow H$ is of the form $f=g \circ h$ where $h$ 
is a map induced by an abstract field isomorphism between $R$ and $L$ and $g$ is an L-semialgebraic isomorphism of groups. In particular $f$ is continuous, and if $R=L$ is the field of real numbers, then $f$ is semialgebraic and therefore a Lie isomorphism of $G$ and $H$.

Proof. Let $R_{1}$ be a real closed field interpretable in $\mathbb{G}$, and let $\sigma$ be a $\mathbb{G}$-definable isomorphism between $G$ and an $R_{1}$-definable group.

By [12, $R$ and $R_{1}$ are isomorphic by an $R$-definable map, say $g_{1}$. It follows that every $R$-definable subset of $R_{1}^{n}, n \in \mathbb{N}$, is also $R_{1}$-definable. Now, if $S \subseteq G^{n}$ is $R$ definable, then $\sigma(S)$ is clearly $R$-definable and therefore $R_{1}$-definable. Hence, $\sigma(S)$ is $\mathbb{G}$-definable and since $\sigma$ is $\mathbb{G}$-definable, we may conclude that $S$ is $\mathbb{G}$-definable, thus proving (1).

It follows from (1) that the map which $g_{1}$ induces on $G$, call it $g_{1}^{*}$, is $\mathbb{G}$-definable. Let $G_{1}=g_{1}^{*}(G)$.

Now let $H$ be a semialgebraic group over a real closed field $L$ and let $f: G \rightarrow H$ be an abstract group isomorphism. Then, as $R_{1}, G_{1}$ and $g_{1}^{*}$ are $\mathbb{G}$-definable, $f$ takes $R_{1}$ to a field $L_{1}$, takes $G_{1}$ to a $\left(L_{1}\right.$-definable) group $H_{1}$ and takes $g_{1}^{*}$ to a group isomorphism $g_{1}^{* *}$ between $H$ and $H_{1}$, where $L_{1}, H_{1}$ and $g_{1}^{* *}$ are all $\mathcal{H}$-definable. We have

$$
g_{1}^{* *}(x)=f g_{1}^{*} f^{-1}(x),
$$

(where of course $f$ acts on tuples coordinatewise and, if the objects are interpretable, on equivalence classes).

Again, by [12, there is a (unique) $L$-definable isomorphism from $L$ to $L_{1}$, call it $g_{2}$. $\left(g_{2}^{*}\right)^{-1}$ is the map which $g_{2}^{-1}$ induces on $H_{1}$. Let $H_{2}=\left(g_{2}^{*}\right)^{-1}\left(H_{1}\right)$. If we now let $\sigma=g_{2}^{-1} \circ f \circ g_{1}$, then $\sigma$ is a field isomorphism between $R$ and $L$ which induces the isomorphism $\sigma^{*}=\left(g_{2}^{*}\right)^{-1} f g_{1}^{*}$ between $G$ and $G_{3}$. But then, by the above,

$$
f(x)=\left(g_{1}^{* *}\right)^{-1} g_{2}^{*} \sigma^{*}(x),
$$

and $\left(g_{1}^{* *}\right)^{-1} g_{2}^{*}$ is a semialgebraic isomorphism between $H_{2}$ and $H$.

Remark 4.9. The proposition above applies not only to definably simple groups. For example, the canonical semidirect product of $\mathbb{R}^{*}$ and $\langle\mathbb{R},+\rangle$ is bi-interpretable with $R$, as is the semidirect product of $S 0(2, \mathbb{R})$ and $\langle\mathbb{C},+\rangle$. Every abstract automorphism of these groups is therefore a Lie automorphism.

We are now ready to finish the proof of Theorem 1.1 .

If $\mathbb{G}$ is bi-interpretable with an algebraically closed field $K$, then in particular it is interpretable in $K$ and therefore by what is known as the Weil-Hrushovski Theorem (see 4.13 in [19]), it is definably isomorphic in $K$ to an algebraic group over $K$. By the bi-interpretability, this isomorphism is $\mathbb{G}$-definable.

Assume now that $G$ is bi-interpretable with a real closed field. First note that we have sufficient information to deduce, as in the remark following Claim 2.23. that the group $G$ is semialgebraically simple and connected. We let $H$ denote its Zariski closure in the algebraic closure $R$. But then $G$ is the semialgebraic connected component of $H(R)$ and so $H$ does not have algebraic subgroups of finite index defined over $R$. If $H$ is not $R$-simple, then it has an algebraic normal nontrivial subgroup $N$ defined over $R$. Since $G$ is semialgebraically simple we have $G \cap N(R)=\{e\}$ and because both groups are normal in $H(R), G$ centralizes $N(R)$. As the centralizer of $N(R)$ in $H$ is algebraic over $R$, we must have $C_{H}(N(R))=H$, i.e. $N \subseteq Z(H)$. The center of $H$ must be finite since otherwise it has a nontrivial 
intersection with $G$. Therefore (see 2.11) $H_{1}=H / Z(H)$, which is itself a linear algebraic group defined over $R$, is centerless. The quotient map, call it $\pi: H \rightarrow H_{1}$, is $\mathbb{G}$-definable and sends $G$ injectively into $H_{1}$, which still does not have algebraic subgroups of finite index defined over $R$. By dimension considerations, $H_{1}$ is the Zariski closure of $\pi(G)$. Since $H_{1}$ is centerless, the above argument shows that it is also $R$-simple.

We now prove a corollary which we promised in Remark 1.2 (b).

Corollary 4.10. Let $G$ be a definably simple, infinite semialgebraic group over a real closed field $R$. Then the following are equivalent:

(i) $\mathbb{G}=\langle G, \cdot\rangle$ is a stable group.

(ii) $G$ is $\mathbb{G}$-definably isomorphic to $H(K)$, where $H$ is an algebraic group and $K$ isomorphic (in $R$ ) to $R(\sqrt{-1})$.

(iii) The Lie algebra of $G$ over $R, \mathcal{L}=L(G)$, is not absolutely simple.

Proof. By Theorem 1.1, if $\mathbb{G}$ is not bi-interpretable with an algebraically closed field, then a real closed field is definable in $\mathbb{G}$ and hence it is unstable. Thus (i) implies (ii).

We assume now that $G$ is semialgebraically isomorphic to an algebraic group $H$ over $R(\sqrt{-1})$. Let $L(G)$ be the Lie algebra of $G$ over $R$ and let $L(H)$ be the Lie algebra of $H$ over $R(\sqrt{-1}) . L(G)$ and $L(H)$ are isomorphic, as Lie algebras over $R$. But $L(H)$, being a Lie algebra over $R(\sqrt{-1})$ cannot be absolutely simple, as a Lie algebra over $R$; its complexification can be shown to be isomorphic, as a Lie algebra over $R(\sqrt{-1})$ to a direct sum of two Lie algebras isomorphic to $L(H)$ (this is usually done in the literature for $\mathbb{R}$ and $\mathbb{C}$ but the same argument works here). Thus (ii) implies (iii). Note that the simplicity of $G$ was not used here.

We now want to show that (iii) implies (i): We let $\mathcal{L}^{\prime}$ be the extension of $\mathcal{L}$ to $K=R(\sqrt{-1})$. Since $\mathcal{L}$ is simple, $\mathcal{L}^{\prime}$ is a semisimple Lie algebra over $K$. If $\mathcal{L}^{\prime}$ is not simple, then, by the decomposition theorem for semisimple Lie algebras, it is the direct sum of simple Lie algebras $L_{1}, \ldots, L_{k}$ over $K$. The projection map from $\mathcal{L}^{\prime}$ onto each of the $L_{i}$ 's is a homomorphism of Lie algebras over $R$ hence, since $\mathcal{L}$ is simple, it is isomorphic as a Lie algebra over $R$ to one of the $L_{i}$ 's. We have shown then, that $\mathcal{L}$ admits a structure of a linear space over $K$ (compatible with its $R$-structure) under which it is a Lie algebra over $K$. We denote by $\mathcal{L}_{R}$ and $\mathcal{L}_{K}$ the two structures. We have $\operatorname{dim} \mathcal{L}_{R}=2 \operatorname{dim} \mathcal{L}_{K}$.

We now repeat in sketch an argument similar to the one given in 14]: Since $\mathcal{L}_{R}$ and $\mathcal{L}_{K}$ are simple Lie algebras over fields of characteristic zero, we have $\operatorname{dim} \mathcal{L}_{R}=$ $\operatorname{dim} \operatorname{Aut}\left(\mathcal{L}_{R}\right)$ and $\operatorname{dim} \mathcal{L}_{K}=\operatorname{dim} \operatorname{Aut}\left(\mathcal{L}_{K}\right)$, where the dimension is taken as vector spaces over $R$ and $K$, respectively. But then, as vector spaces over $R$, we have $\operatorname{Aut}\left(\mathcal{L}_{R}\right) \subseteq \operatorname{Aut}\left(\mathcal{L}_{K}\right)$ and the two have the same dimension.

Since $\operatorname{Aut}\left(\mathcal{L}_{R}\right)$ and $\operatorname{Aut}\left(\mathcal{L}_{K}\right)$ are definable in $R$ the group $\operatorname{Aut}\left(\mathcal{L}_{R}\right)$ has finite index in $\operatorname{Aut}\left(\mathcal{L}_{K}\right)$. As is pointed out in [14], $G$ is isomorphic (in $R$ ) to $G_{1}$, the semialgebraic connected component of $\operatorname{Aut}\left(\mathcal{L}_{R}\right)$, which is thus also the semialgebraic connected component of $\operatorname{Aut}\left(\mathcal{L}_{K}\right)$. However, since $\mathcal{L}_{K}$ is a Lie algebra over $K$ its automorphism group is an algebraic group over $K$, and therefore its semialgebraic connected component is the irreducible component containing the identity, hence an algebraic group over $K$. We showed then that $G$ is (abstractly) isomorphic to an algebraic group and therefore must be stable. 
We conclude with an observation that the field which we interpreted in $\mathbb{G}$ can be chosen to be interpretable without parameters (other than the ones used to define $G)$.

There are two cases to consider. Assume first that the field we defined is real closed. Then there are formulas $\phi(x, y), \phi_{+}(\bar{x}, y)$ and $\phi .(\bar{x}, y)$ in the group language, such that for every $a \in G, \phi(G, a)$ is a real closed field, call it $k_{a}$, whose operations are given by $\phi_{+}(\bar{x}, a)$ and $\phi .(\bar{x}, a)$. By [12], for every $a_{1}, a_{2}$ there is an $R$-definable isomorphism between $k_{a_{1}}$ and $k_{a_{2}}$. By o-minimality, this isomorphism is unique, denote it by $\sigma_{a_{1}, a_{2}}$. Using the compactness theorem, we can find finitely many formulas $\psi_{i}\left(x_{1}, x_{2}, y_{1}, y_{2}\right)$ such that every $\sigma_{a_{1}, a_{2}}$ is given by one of the $\psi_{i}$ 's.

It is now easy to show that one can interpret a field $k$ isomorphic to the $k_{a}$ 's without parameters. Its universe is obtained as the quotient of the $(x, y)$ 's by the equivalence relation $\left(x_{1}, y_{1}\right) \sim\left(x_{2}, y_{2}\right)$ if and only if $\sigma_{y_{1}, y_{2}}\left(x_{1}\right)=x_{2}$. Its operations are induced from the field operations of the $k_{a}$ 's. $k$ is interpretable without parameters.

If $\mathbb{G}$ is bi-interpretable with an algebraically closed field $K$, then one can repeat the same argument with $k$ replaced by $K$, using the isomorphism theorem of Poizat (4.15 in [19]).

Remark 4.11. Even though $K$ may be interpreted in $\mathbb{G}$ without parameters, $K$ and $\mathbb{G}$ are not bi-interpretable without parameters. That is, for otherwise any definable automorphism of the group $G$ would give rise to a definable automorphism of $K$, but $G$ has many definable automorphisms (namely inner ones) while $K$ has none.

Added in proof. We recently realized that, in the " $R$-anisotropic case" (Section 2.3.1), one can replace our current argument which uses the structure of compact Lie groups by a model theoretic argument.

After observing that $G$ in this case is closed and bounded in $G L(n, R)$ we proceed as follows: We first prove that in $\mathbb{G}$ one can define uniformly a basis for the group topology. Indeed, if we take $h$ in $G$, then, by [3.13, a finite product of $h^{-1} h^{G}$ contains an open neighborhood of $e$. Because $G$ is closed and bounded, as $h$ approaches $e$ these neighborhoods get as small as we wish.

Using this topology we can now define in $\mathbb{G}$ a set $D \subseteq G$ whose dimension is 1. Moreover, we can definably in $\mathbb{G}$ equip $D$ with a linear ordering such that the structure induced on $D$ by $\mathbb{G}$, call it $\mathcal{D}$, is o-minimal. We then can use the Trichotomy theorem for o-minimal structures (see [15]) to deduce that a real closed field is definable in $\mathcal{D}$ and therefore in $\mathbb{G}$.

\section{REFERENCES}

1. O. Belegradek, On mutual interpretation of a ring $R$ and the group $U T_{n}(R)$, preprint.

2. A. Borel, Linear Algebraic Groups, 2nd ed., Graduate Texts in Math., 126 (Spinger-Verlag, 1991). MR 92d:20001

3. A. Borel and J. Tits, Homomorphismes "abstraits" de groupes algébraic simples, Annals of Math. 97 (1973), 499-571. MR 47:5134

4. A. Borovik and A. Nesin, Groups of finite Morley rank, (The Clarendon Press, Oxford University Press, New York, 1994). MR 96c:20004

5. L. van den Dries, O-minimal structures, Logic: From foundations to applications, (ed: Hodges, Steinhorn, Truss), Oxford University Press, 1996, 137-185. MR 98b:03053

6. L. van den Dries Tame topology and o-minimal structures, Cambridge University Press, Cambridge, 1998. MR 99j:03001

7. H. Freudenthal, Die Topologie der Lieschen gruppen als algebraisches Phänomenä. I, Ann. of Math 42 (1941), 1051-1074. Erratum ibid. 47 (1946), 829-830. MR 3:198a 
8. W. Hodges, Model theory, Encyclopedia of Mathematics and its Applications, vol. 42 (Cambridge University Press, Cambridge, 1993). MR 94e:03002

9. E. Hrushovski and A. Pillay, Groups definable in local fields and pseudo-finite fields, Israel Journal of Mathematics 85 (1994), 203-262. MR 95f:12015

10. D. Marker, Semialgebraic expansions of $\mathbb{C}$, Trans. Amer. Math. Soc. 320 (1990), 581-592. MR 90k:03034

11. A. Nesin and A. Pillay, Some model theory of compact Lie groups, Trans. Amer. Math. Soc. 326 (1991), 453-463. MR 91j:03038

12. M. Otero, Y. Peterzil and A. Pillay, Groups and rings definable in o-minimal expansions of real closed fields, Bull. London Math. Soc. 28 (1993) 7-14. MR 96i:12006

13. Y. Peterzil, Constructing a group interval in o-minimal structures, J. of Pure and Applied Algebra 94 (1994), 85-100. MR 95h:03085

14. Y. Peterzil, A. Pillay and S. Starchenko, Definably simple groups in o-minimal structures, Trans. Amer. Math. Soc. 352 (2000), 4397-4419. CMP 99:17

15. Y. Peterzil and S. Starchenko, A trichotomy theorem for o-minimal structures, Proceedings of the London Mathematical Society (3) 77 (1998), 481-523. MR 2000b:03123

16. A. Pillay, On groups definable in o-minimal structures, J. of Pure and Applied Algebra 53 (1988), 239-255. MR 59i:03069

17. A. Pillay, An application of model theory to real and p-adic algebraic groups, J. of Algebra 126 (1989), 139-146. MR 90m:03061

18. V. Platonov and A. Rapinchuk, Algebraic groups and number theory, Pure and Applied Mathematics, v. 139., Academic Press, Inc. MR 95b:11039

19. B. Poizat, Group Stables, (Nur al-Mantiq wal-Ma'rifah, 1987). MR 89b:03056

20. A. Strzebonski, Euler characteristic in semialgebraic and other o-minimal groups, J. Pure Appl. Algebra 96 (1994), 173-201. MR 95j:03067

21. J. Tits, Homomorphismes et automorphismes "abstraits" de groupes algébriques et arithmétique, Actes du Congrès Internat. des Math., Tome 2, 1970, 349-355. MR 55:5760

22. B. Weisfeiler, On abstract homomorphisms of anisotropic algebraic groups over real-closed fields, Journal of Algebra 60 (1979), 485-519. MR 80k:20045

Department of Mathematics and Computer Science, Haifa University, Haifa, Israel

E-mail address: kobi@mathcs2.haifa.ac.il

Department of Mathematics, University of Illinois at Urbana-Champaign, 1409 W. Green St., Urbana, Illinois 61801

E-mail address: pillay@math.uiuc.edu

Department of Mathematics, University of Notre Dame, CCMB, Notre Dame, Indiana 46556

E-mail address: starchenko.1@nd.edu 